\title{
Nano drug delivery systems improve metastatic breast cancer therapy
}

https://doi.org/10.1515/mr-2021-0011

Received April 29, 2021; accepted August 3, 2021;

published online October 21, 2021

\begin{abstract}
Despite continual progress in the technologies and regimens for cancer therapy, the treatment outcome of fatal metastatic breast cancer is far from satisfactory. Encouragingly, nanotechnology has emerged as a valuable tool to optimize drug delivery process in cancer therapy via preventing the cargos from degradation, improving the tumor-targeting efficiency, enhancing therapeutic agents' retention in specific sites, and controlling drug release. In the last decade, several mechanisms of suppressing tumor metastasis by functional nano drug delivery systems (NDDSs) have been revealed and a guidance for the rational design of anti-metastasis NDDSs is summarized, which consist of three aspects: optimization of physiochemical properties, tumor microenvironment remodeling, and biomimetic strategies. A series of medicinal functional biomaterials and anti-metastatic breast cancer NDDSs constructed by our team are introduced in this review. It is hoped that better anti-metastasis strategies can be inspired and applied in clinic.
\end{abstract}

Keywords: breast cancer; drug delivery; metastasis; nanotechnology; stimuli-responsive.

Runqi Zhu and Tianqun Lang are contributed equally to this work.

*Corresponding authors: Prof. Qi Yin and Prof. Yaping Li, State Key Laboratory of Drug Research \& Center of Pharmaceutics, Shanghai Institute of Materia Medica, Chinese Academy of Sciences, 501 Haike Road, Shanghai 201203, China, E-mail: qyin@simm.ac.cn (Q Yin) ypli@simm.ac.cn (Y Li). https://orcid.org/0000-0002-0574-6966 (Y Li)

Runqi Zhu, State Key Laboratory of Drug Research \& Center of Pharmaceutics, Shanghai Institute of Materia Medica, Chinese Academy of Sciences, Shanghai, China; and University of Chinese Academy of Sciences, Beijing, China

Tianqun Lang, State Key Laboratory of Drug Research \& Center of Pharmaceutics, Shanghai Institute of Materia Medica, Chinese Academy of Sciences, Shanghai, China; University of Chinese Academy of Sciences, Beijing, China; and Yantai Key Laboratory of Nanomedicine \& Advanced Preparations, Yantai Institute of Materia Medica, Yantai, Shandong Province, China

\section{Introduction}

With the highest morbidity in women, breast cancer has become a growing health threat world-wide [1]. Metastasis is the greatest contributor ( $>90 \%$ ) to the high mortality of breast cancer patients [2, 3]. Although breast cancer diagnosed at the early stage can be cured, metastasis into distant organs at advanced stages is still fatal. The most common sites where metastasis occurs are the lung, bone, liver, and brain. Particularly, lung metastasis directly causes 60-70\% of death of breast cancer patients [4, 5]. Despite continual progress in cancer therapy and a few patients with metastatic cancer cured by current therapeutic modalities, the treatment of metastatic breast cancer is far from satisfactory. When analyzing metastatic breast cancer clinical data over the past 30 years, there is no sustained evidence indicating increasing patient survival, presenting a dismal picture [2]. Therefore, it is urgent to elucidate the etiology and molecular mechanisms of metastatic breast cancer and find out efficient therapy methods to target metastasis.

Molecular targeting therapy, immunotherapy and combination therapy have greatly improved therapeutic outcomes of metastatic cancer, compared with traditional cytotoxic agents. However, the highly dispersed metastasis foci and solid tissue barriers of primary tumors aggravate the predicament of insufficient drug concentrations in the tumors $[2,6,7]$. To increase the accumulation of active drugs at target sites, new approaches combining engineering, medical and oncology may become promising choices. Nanotechnology has emerged as a valuable tool to optimize drug delivery process via preventing the cargos from degradation, improving the targeting activity of delivery, controlling drug release and enhancing therapeutic agents' retention in specific sites [7, 8]. Taking advantage of the enhanced permeability and retention (EPR) effect, versatile active targeting approaches and programmed cargo release strategies, nano drug delivery systems (NDDSs) have attracted increasing attentions as a promising treatment alternative to conventional modalities for metastatic cancer [7-9]. A variety of biological, organic and inorganic materials with distinct properties have been exploited to fabricate nanoscale vehicles. 
Table 1: Summary of NDDS-based marketed products to treat cancer.

\begin{tabular}{|c|c|c|c|c|c|c|}
\hline Product & Type of NDDS & $\begin{array}{l}\text { Active pharmaceutical } \\
\text { ingredients }\end{array}$ & Indication & Status & $\begin{array}{r}\text { Year of first } \\
\text { introduced }\end{array}$ & References \\
\hline Doxil $^{\circledR}$ & Liposome & Doxorubicin & $\begin{array}{l}\text { HIV-related Kaposi sarcoma, } \\
\text { ovarian cancer and multiple } \\
\text { myeloma }\end{array}$ & Approved by FDA & 1995 & [8] \\
\hline DaunoXome $^{\circledR}$ & Liposome & Daunorubicin & $\begin{array}{l}\text { AIDS-related Kaposi's sarcoma } \\
\text { and metastatic ovarian cancer }\end{array}$ & Approved by FDA & 1996 & [8] \\
\hline Marqibo $^{\circledR}$ & Liposome & Vincristine sulfate & Acute lymphoid leukemia & Approved by FDA & 2012 & [8] \\
\hline Onivyde ${ }^{\circledR}$ & Liposome & Irinotecan & Pancreatic cancer & Approved by FDA & 2015 & [8] \\
\hline Myocet $^{\circledR}$ & Liposome & Doxorubicin & Breast cancer & $\begin{array}{l}\text { Approved in } \\
\text { Europe and } \\
\text { Canada }\end{array}$ & 2000 & [8] \\
\hline Abraxane $^{\circledR}$ & $\begin{array}{l}\text { Albumin } \\
\text { nanoparticle }\end{array}$ & Paclitaxel & $\begin{array}{l}\text { Breast, lung and pancreatic } \\
\text { cancer }\end{array}$ & Approved by FDA & 2005 & [8] \\
\hline Genexol-PM $^{\circledR}$ & $\begin{array}{l}\text { Polymeric } \\
\text { micelle }\end{array}$ & Paclitaxel & $\begin{array}{l}\text { Breast cancer and non-small cell } \\
\text { lung cancer }\end{array}$ & Approved in Korea & 2007 & [8] \\
\hline Eligard $^{\circledR}$ & $\begin{array}{l}\text { Polymeric } \\
\text { nanoparticle }\end{array}$ & Leuprolide acetate & Prostate cancer & Approved by FDA & 2002 & [11] \\
\hline Ontak $^{\circledR}$ & Fusion protein & Denileukin diftitox & Cutaneous T-cell lymphoma & Approved by FDA & 1999 & [10] \\
\hline Vyxeos $^{\circledR}$ & Liposome & $\begin{array}{l}\text { Daunorubicin and } \\
\text { Cytarabine }\end{array}$ & Acute myeloid leukemia & Approved by FDA & 2017 & [10] \\
\hline Mepact $^{\circledR}$ & Liposome & Mifamurtide & Osteosarcoma & $\begin{array}{l}\text { Approved in } \\
\text { Europe }\end{array}$ & 2009 & [10] \\
\hline DepoCyte ${ }^{\circledR}$ & Liposome & Cytarabine & $\begin{array}{l}\text { Lymphomas or leukemia with } \\
\text { meningeal spread add neoplastic } \\
\text { meningitis }\end{array}$ & Approved by FDA & 2005 & [10] \\
\hline Lipusu $^{\circledR}$ & Liposome & Paclitaxel & Breast and ovarian cancer & Approved in China & 2003 & [15] \\
\hline SMANCS $^{\circledR}$ & $\begin{array}{l}\text { Polymer } \\
\text { conjugate }\end{array}$ & $\begin{array}{l}\text { Styrene maleic acid- } \\
\text { Neocarzinostatin }\end{array}$ & Liver and renal cancer & Approved in Japan & 1993 & [8] \\
\hline Oncaspar ${ }^{\circledR}$ & $\begin{array}{l}\text { Polymer } \\
\text { conjugate }\end{array}$ & PEG-L-asparaginase & $\begin{array}{l}\text { Acute lymphocytic leukemia, } \\
\text { lymphoblastic leukemia and } \\
\text { lymphoma }\end{array}$ & Approved by FDA & 1994 & [10] \\
\hline SYLATRON $^{\circledR}$ & $\begin{array}{l}\text { Polymer } \\
\text { conjugate }\end{array}$ & PEG-interferon $\alpha-2 b$ & $\begin{array}{l}\text { Melanoma with microscopic or } \\
\text { gross nodal }\end{array}$ & Approved by FDA & 2002 & [16] \\
\hline
\end{tabular}

AIDS: acquired immunodeficiency syndrome; FDA: Food and Drug Administration; HIV: human immunodeficiency virus; NDDS: nano drug delivery system; PEG: polyethylene glycol.

In the last 30 years, the clinic applications of NDDSs for cancer treatment has achieved notable success. Since Food and Drug Administration (FDA) approved the first nanomedicine Doxil ${ }^{\circledR}$ [the doxorubicin (DOX)-loaded unilamellar polyethylene glycol (PEG)-modified -liposome] for treating cancers such as metastatic breast cancer, ovarian cancer and Kaposi's sarcoma-associated with acquired immune deficiency syndrome, 16 forms of NDDS-mediated therapies for cancer treatment have appeared on the market globally (Table 1) [10]. Among these products, liposomes such as Marqibo ${ }^{\circledR}$ (vincristine) and Onivyde ${ }^{\circledR}$ (irinotecan) are the most widely used formulations in clinic due to good-biocompatibility and well-developed manufacturing process, achieving slower degradation and minimal side effects of cytotoxic agents [11]. Other nano formulations including protein-based nanoparticles
[Abraxane ${ }^{\circledR}$ (Paclitaxel) and Ontak ${ }^{\circledR}$ (diphtheria)], polymeric micelles [Genexol-PM ${ }^{\circledR}$ (paclitaxel) and Eligard ${ }^{\circledR}$ (leuprolide)], and polymer-drug conjugates [SMANCS $^{\circledR}$ (neocarzinostatin), Oncaspar ${ }^{\circledR}$ (asparaginase), and SYLATRON $^{\circledR}$ (interferon $\left.\left.\alpha-2 b\right)\right]$ only take a small part (7 in 16) of approved nanomedicines [11]. As publicized on the website (https://clinicaltrials.gov/) of National Institutes of Health $(\mathrm{NIH})$, there are 110 nanotechnology-based cancer treatments undergoing clinical trials in 2020. Billions of dollars and thousands of scientists have been attracted in this rapid-developing field. The clinical translation of nanomedicine is associated with complex and time-consuming process. Compared to conventional formulations, nanomedicine faces greater challenges in drug delivering system design, evaluation system establishment, up-scaled manufacturing, and regulatory barriers [12, 13]. Although 
approved NDDSs for cancer treatment are still limited in number and formulation, new therapeutic agents beyond cytotoxic drugs (e.g., siRNA, miRNA and kinase inhibitors) and tumor-imaging agents have been included in developing versatile nanomedicine for clinical purpose [14]. More types of promising NDDSs including gold nanoparticles [NU-0129 for treating recurrent glioblastoma or gliosarcoma undergoing surgery (NCT03020017)] and dendrimers [AZD0466 for treating advanced solid tumors (NCT04214093)] are undergoing clinical trials.

Since NDDSs are versatile platforms for combining multiple therapeutic functions with targeting, detection and particle trafficking capabilities, they hold great promise for diagnosis and treatment of metastatic cancer $[7,8]$. In the last decade, our team have focused on nanotechnologybased drug delivery systems for the therapy of metastatic breast cancer. The mechanisms of suppressing tumor metastasis by multiple functional NDDSs have been revealed [17]. Based on these understandings, a guidance consisting of three approaches including stimuli-responsive optimization of physiochemical properties, tumor microenvironment remodeling and biomimetic strategies for the rational design of anti-metastasis NDDSs have been summarized [17-20]. Based on the unique understanding of therapeutic targets that are responsible for metastases, our team have designed and synthesized a series of medicinal functional biomaterials and constructed well-characterized drug delivery systems which successfully inhibited metastatic breast cancer on animal models [21-31].

In this review, firstly, the basic principles of combating metastatic breast cancer by nanotechnology are outlined. Then several representative research about well-designed anti-metastasis NDDSs with bright prospects are introduced. Finally, challenges and opportunities of treating metastatic cancer with nanotechnology-based tools are discussed. It is hoped that better choices for antimetastasis therapy via NDDSs will be found and more valuable research will be inspired.

\section{Principles for the rational design of anti-metastasis NDDSs}

Metastasis is the spread of tumor cells from a primary site to other parts of the body. Over the past 20 years, significant progress has been made in revealing the biological principles of the metastasis process. Metastasis, as a complicated multi-step process, starts from the dissemination of primary cancer cells during which epithelial-mesenchymal transition (EMT) plays an important role $[6,32]$. Then the invasion of cancer cells into the vasculature provides access to the travel to distant sites. The extravasation of circulating tumor cells (CTCs) through the endothelial wall and the development of metastatic colonies represents the final and most deadly phase in metastasis [2, 6]. CTCs, like tumor seeds, grow on the soil provided by metastatic microenvironment. The interaction between cancer cells and various components in specific niches including extracellular matrix (ECM), stromal cells, endothelial cells, cancerassociated fibroblasts (CAFs), and immune cells support the growth of metastatic foci $[6,33,34]$. Besides tumor signaling pathways involved in metastatic colonization, newly emerging targets in metastatic microenvironment such as chemokines, tumor-associated macrophages (TAMs), immune checkpoints, and tumor-derived exosomes, etc. provide great outlook in improving outcomes of malignant cancer therapy $[2,35]$.

The understanding of pathogenic mechanisms and influence factors of metastasis lay the foundation for the design of anti-metastasis NDDSs. Although NDDSs have shown potentials in treating cancer, crucial challenges are existing in the avenue toward efficient nanotechnologybased therapy of metastatic breast cancer. Wellvascularized primary tumors with large sizes are favorable for the EPR effect to work, allowing specific accumulation of nanoparticles in tumor tissues [7, 17, 33, 34]. However, as small disseminated malignant cohorts, secondary tumors are poor-vascularized and highly dispersed to multiple organs, which make it more difficult for therapeutic agents to reach cancer cells in metastatic sites than those in primary tumors [6, 17]. Biological barriers also significantly exacerbate the inadequacy of the penetration of nanoparticles in these metastases [18]. Therefore, overcoming these obstacles to increase the intra-tumoral accumulation, intra-tumoral penetration, and cellular uptake of drugs is the first priority to efficiently suppress the growth of primary and metastatic tumors.

In spite of the obstacles in tumor tissues that prevent NDDSs from reaching the target sites, certain special factors in the tumor microenvironment (TME) provide opportunities for the design of anti-metastasis NDDSs. Irregular angiogenesis in the fast-growing tumors leads to insufficient nutrition and oxygen provision for tumor growth, making the tumor cores hypoxia [36]. The accumulation of acidic metabolites at the tumor site after glycolysis results in slight acidity (pH 6.5-7.2) in the tumor interstitium [8]. The up-regulated enzymes at the tumor site (e.g., protease, hyaluronidase and legumain) are another important type of endogenous stimuli, which has important effects on tumor growth, angiogenesis, tumor cell invasion, and 
metastasis [30, 37-39]. Besides, the concentration of glutathione (GSH) in tumor cells is 100-1000 times and 100 times higher than that in blood and normal tissues, respectively [40]. These endogenous factors provide conditions for triggering the changes in the physiochemical properties of NDDSs.

There follow three major strategies to overcome the barriers for the drugs to reach their action sites: (1) Control the transport behaviors by optimizing physiochemical properties; (2) Remodel tumor microenvironment; (3) Biomimetic strategies.

\section{Control the transport behaviors by optimizing physiochemical properties}

In order to achieve long blood-circulation and overcome the solid physical barriers in metastatic lesions, properties of NDDSs including the size, the surface charge, the shape and surface modification should be carefully regulated.

\section{Optimize the particle size}

It is a general agreement that smaller nanoparticles (10$100 \mathrm{~nm}$ ) can penetrate more easily into tumor tissues through leaky tumor vasculature [33]. Wang et al. [41] synthesized 7-ethyl-10-hydroxylcamptothecin (SN38) prodrug micelles with various sizes ranging from 20 to $300 \mathrm{~nm}$ to study the optimal particle size for cancer drug delivery. They found that the balance between drug accumulation and tumor penetration was crucial. The $100-160 \mathrm{~nm}$ micelles showed optimal tumor accumulation but no superior therapeutic outcome over the $30 \mathrm{~nm}$ ones due to poor tumor penetration [41]. In general, nanoparticles averaging approximately $100 \mathrm{~nm}$ showed better accumulation and penetration in target sites and long-lasting circulation in the blood [41-43].

\section{Optimize the surface charge}

The positive surface charge is favorable for cellular uptake, but positively charged NDDSs are easily detected and cleared by the mononuclear phagocyte system [33]. To solve this key problem, Lang et al. [44] synthesized an acidsensitive polymer, poly((1,4-butanediol)-diacrylate- $b-N, N$ diisopropylethylenediamine)-polyethyleneimine (BD-PEI), and a matrix metalloproteinase (MMP)-responsive polymer, poly((1,4-butanediol)-diacrylate- $b$ - $N, N$-diisopropylethylenediamine)-peptide-polyethylene glycol (BD-peptide$\mathrm{PEG})$. Then the tumor microenvironment-responsive micelles were prepared by BD-PEI and BD-peptide-PEG to deliver docetaxel (DTX). When the micelles reached tumor tissues, their particle sizes turned smaller and their zeta potentials went higher, leading to more DTX being delivered into tumor cells. The acid-sensitive materials ensured the quick release of drugs in tumor cells, thereby inhibited the growth of breast cancer in situ and pulmonary metastasis. This system revealed that increasing the uptake of nano-drugs into tumor cells and the rate of intracellular drug release could effectively inhibit breast cancer with lung metastasis [44].

\section{Optimize the shape}

The transport behaviors of nanosystems depend on their shapes. Zhang et al. [45] reported that elongated polystyrene (PS) nanoparticles with higher aspect ratios at fixed volumes showed better uptake rates in the evaluated 3-dimension (3D) spheroid model than in the 2-dimension (2D) cell monolayer culture model. Similarly, a study by Dias et al. [46] indicated that gold core-mesoporous silica shell nanorods displayed an increased intracellular migration and uptake in 2D cell cultures than sphere nanoparticles. The shape of nanoparticles should be rationally chosen depending on the application requirements. Kolhar et al. [47] reported that rod-shaped nanoparticles displaying blood vessel-targeting ligands exhibited higher specificity under flow compared with their spherical counterparts. According to mathematical modeling analysis, this enhanced targeting effects resulted from increasing polyvalent interactions of rod-shaped nanoparticles that favor adhesion and entropic losses, which fought against shear-induced binding reduce. In vivo experiments showed that shape-induced enhancement of vascular targeting of rod-shaped nanoparticles could be exploited to improve organ specificity of drug delivery systems [47]. Moreover, Geng et al. [48] found that filomicelles persisted in the circulation 10 times longer than their spherical counterparts. Based on the hypothesis that rod-shaped nanoparticles increased the accumulation in specific organs like lungs and improved the internalization by cancer cells after intravenous administration, He et al. [49] reported the design and construction of succinobucol-, a selective inhibitor of vascular cell adhesion molecule-1 (VCAM-1), loaded $\mathrm{pH}$-responsive wormlike micelles, termed as PWM, with sequential targeting capability. Employing the unique shape, PWM were firstly efficiently transported to lung tissues, exhibiting specific targeting capacity to metastatic nodules compared with free succinobucol. After releasing succinobucol in cancer cells, the expression of VCAM-1 and cell-cell binding were inhibited, contributing 
to preventing lung metastases of $4 \mathrm{~T} 1$ breast cancer model [49]. With intriguing biological properties, nanorods and wormlike nanobodies ought to be paid more attentions to for developing potential nanovesicles for anti-metastatic therapy.

\section{Modify the surface}

Surface modification is another key factor impacting the in vivo behaviors of nanoparticles. Various approaches to endowing NDDSs with specific biological functions include antibodies or ligands decoration, biomimetic materials modification, and "bulldozer" molecules (destructing solid tumor defense with or without exogenous supply) conjugation, etc. $[18,33,34]$ Ligands modification is one of the most widely-used strategies for NDDSs design, with enlarging targeting moiety arsenal including aptamers, peptides, and small molecules [50]. Folic acid is a representative small molecular targeting ligand used in designing target NDDSs. Folic acid exhibits advantages over other ligands on cost, immunogenicity, the size, and accessibility of chemical modification [51]. Wang et al. [52] improved the targeted drug delivery by conjugating folic acid on PEGylatedphospholipid-coated mesoporous silica nanoparticles (MSNs). The non-specific binding and aggregation was reduced, and the cellular uptake in the folate receptorsexpressing cancer cell line was enhanced [52]. To date, the cell-penetrating peptide family has been one kind of promising candidates that efficiently enhance the tumor accumulation and penetration of NDDSs. As integrin-specific ligands, these peptides could enhance the cellular uptake of nanomaterials and affect cancer migration and invasion by interacting with integrins on cancer and angiogenic endothelial cells [34, 53, 54]. Peptide ligands with small sizes and short chains are more stable and economic compared to antibodies. Wang et al. [55] reported a tumor aciditytriggered nanoparticle modified with the cell penetrating peptide iRGD for cancer therapy. The intra-tumoral diffusion of payloads in this NDDS was significantly promoted under the combination of acidity-triggered carrier dissociation, reactive oxygen species (ROS) generation upon near-infrared (NIR) laser irradiation, and iRGD-mediated tumor penetration and cellular uptake [55]. Hu et al. [53] constructed a cabazitaxel-loaded nanovesicle with 1,2-distearoyl-sn-glycero-3-phosphoethanolamine- $N$-methoxy-polyethylene glycol-2000 (DSPE-PEG2000), which was modified with the cell-penetrating D-oligoarginine peptide (R9). Compared with the non-targeting carriers, the R9-modified vesicle infiltrated deeply into primary tumors as well as nearby lymph nodes. Based on characteristics mentioned above, these nanovesicles could remarkably inhibit tumor growth in situ and the formation of lymphatic or lung metastases [53]. This delivery platform provided a new option for the lymphatic metastases-targeting drug delivery.

In conclusion, the regulation of the physicochemical properties of NDDSs can be programmed via stimuliresponsive moieties, which are sensitive to the environment variations in vivo (e.g., $\mathrm{pH}$, redox or enzymes). Appropriate properties and surface modifications would improve the drug accumulation, penetration, and release in primary tumors and metastasis sites. Meanwhile, responsiveness to external stimuli (e.g., light, ultrasound or radiation) ensure spatio-temporally specific treatment of primary tumors and metastasis. These characters cooperate together to inhibit metastasis in distant organs $[55,56]$.

\section{Remodel tumor microenvironment}

Solid tumors present severe defense against deeppenetration drug delivery [6]. Uncontrollable rapid proliferation of cancer cells destructs the normal structures of the local tissues. Aberrant vasculature in the solid tumor is quite tortuous, heterogeneous and chaotic, which negatively affects the distribution and penetration of nanomedicine [57]. Collapsed lymphatic vessels and deformed vasculature in tumors lead to insufficient tissue interstitial fluid convection and interstitial hypertension [34, 58]. Abnormally proliferating tumor cells and stromal cells will cause excessive production and density of ECM, contributing to increased interstitial fluid pressure (IFP) [58-60]. As a result, IFP in solid tumors ranges from 5 to $40 \mathrm{mmHg}$, much higher than $3 \mathrm{mmHg}$ in normal tissue [34]. CAFs and TAMs play important roles in shaping the immune suppression microenvironment and hinder the permeation of nanoparticles [60]. Therefore, remodeling the tumor microenvironment (e.g., vascular normalization, ECM degradation, reducing the percentage of CAFs) can be an effective strategy for promoting the penetration of NDDSs. The angiotensin II receptor antagonist losartan is a potential reagent for CAFs inhibiting, thus subsequently decreases the level of cytokines in the tumor microenvironment. CAFs inhibition also prevents EMT and eliminates metastatic cancer cells. $\mathrm{Hu}$ and co-workers [61] designed and synthesized a series of amphiphilic polypeptides. The materials with good losartan-loading capacity were selected to prepare injectable hydrogels for the local delivery of losartan. After injected in the breast tumor sites, the drug-loaded hydrogels exhibited obvious prolonged retention, continuously releasing losartan to inhibit the activation and function of CAFs, and significantly 
destructed tumor matrix. Subsequently, DOX-loaded liposomes were intravenously injected. Due to the damage of tumor barriers, the accumulation and penetration of DOX in tumor tissues were largely ameliorated. The effect on inhibiting tumor growth and tumor metastasis confirmed that chemotherapy can be significantly improved by adjusting the tumor microenvironment [61].

Besides CAFs inhibitors, NIR irradiation is widely applied as an efficient and controllable approach to immediate increase of vascular permeability. Photodynamic therapy and photothermal therapy dependent on NIR irradiation were found to largely favor tumor treatment through increasing the permeability of tumor tissues, enhancing the cellular uptake of drugs and stimulating the immune response against tumors [27, 28, 62]. Meanwhile, photosensitizers under irradiation can not only generate fluorescence for tracking NDDSs but also convert light into heat to trigger the precise drug release at target sites. Our group has reported several NIR-activated NDDSs for the treatment of metastatic breast cancer, which will be introduced in details later.

\section{Biomimetic strategies}

Natural organisms have specific biological properties such as good biocompatibility and low immunogenicity, which would be inherited by biomimetic NDDSs to favor the antimetastasis treatment [20]. As typical representatives with the long blood circulation ability, red blood cell (RBC) membrane-coated nanovesicles push back the frontiers of the biomimetic drug delivery field. In addition, monocytes/ macrophages can be recruited into tumor sites, and cancer cell membrane coating endows drug delivery systems with the natural homotypic tumor-homing capacity [63, 64]. Inspired by these advantages, certain biomimetic strategies have been exploited to develop NDDSs for metastatic cancer therapy, which will be discussed later.

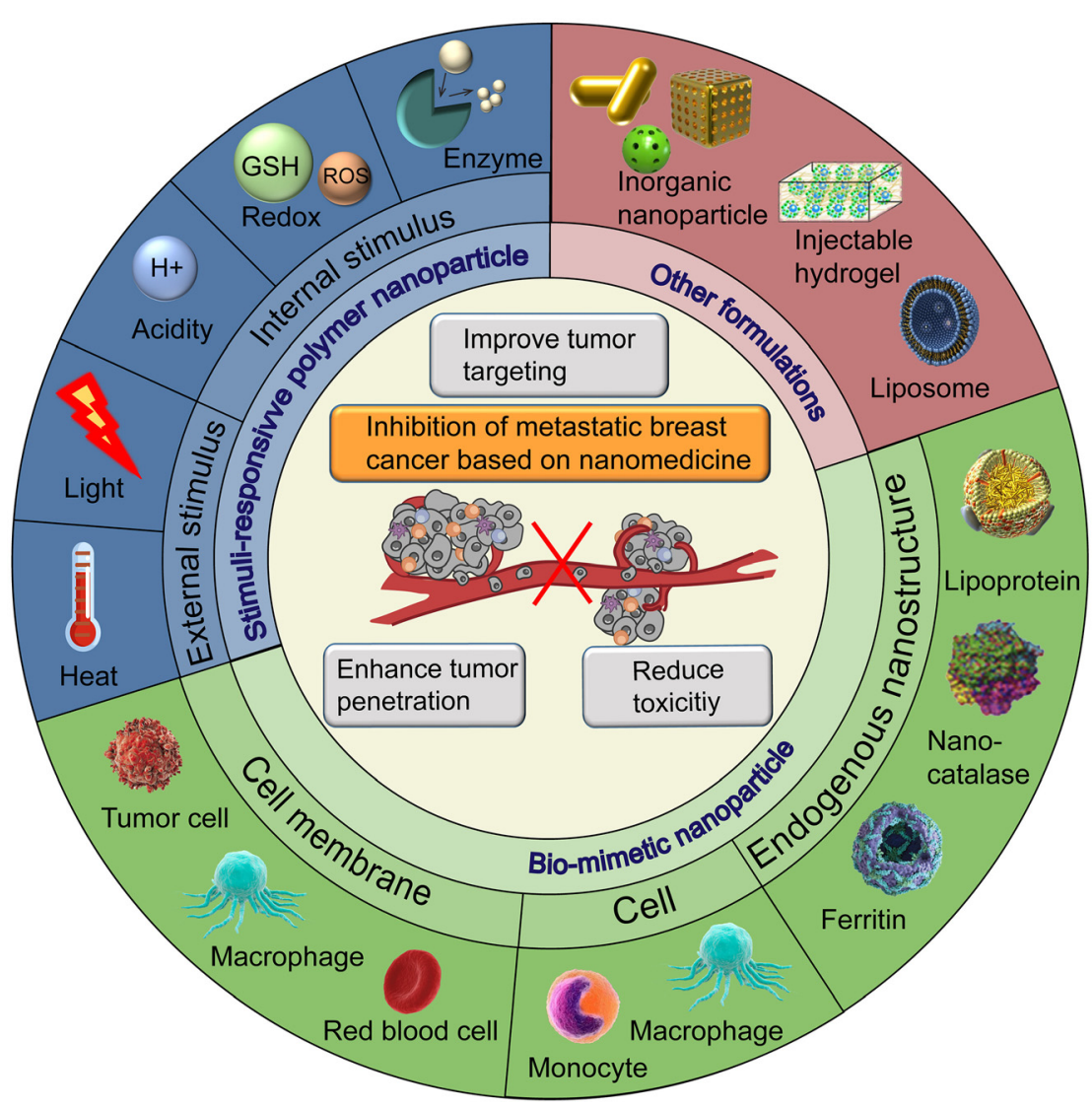

Figure 1: Inhibition of metastatic breast cancer by versatile NDDSs. A series of NDDSs based on different materials, including stimuliresponsive polymers, cell-derived and other endogenous components, lipids and inorganic nanoparticles, have been constructed. Stimuliresponsive polymers have been synthesized to prepare drug delivery vehicles with the capability of controlling drug release, which are encouraging for overcoming physiological barriers and targeting delivery. Light-responsive NDDSs have been applied for the photothermal or photodynamic therapy against breast cancer. Biomimetic strategies, including coating nanoparticles with cell membranes, loading nanoparticles into living cells or fabricating nanoparticles using endogenous materials, endow NDDSs with certain biological functions and good biocompatibility. GSH: Glutathione NDDS: Nano drug delivery systems ROS: Reactive oxygen species redox: Reduction/oxidation. 
Table 2: Summary of representative work on NDDSs-based anti-metastasis therapy.

\begin{tabular}{|c|c|c|c|c|}
\hline $\begin{array}{l}\text { NDDSs for treat- } \\
\text { ing metastatic } \\
\text { breast cancer }\end{array}$ & Property & Design & Key advances & References \\
\hline
\end{tabular}

\begin{tabular}{|c|c|c|}
\hline \multirow{3}{*}{\multicolumn{2}{|c|}{$\begin{array}{l}\text { Stimuli-respon- } \\
\text { sive polymeric } \\
\text { NDDSs }\end{array}$}} & $\begin{array}{l}\text { Nanocomplex containing PTX and siRNA } \\
\text { (siAkt) }\end{array}$ \\
\hline & & $\begin{array}{l}\text { Micelleplex containing the cisplatin pro- } \\
\text { drug and siRNA targeting the NF- } \mathrm{KB} \\
\text { pathway } \\
\text { Host-guest nanosystem containing } \\
\text { succinobucol }\end{array}$ \\
\hline & & $\begin{array}{l}\mathrm{PTX} / \text { calcitriol-co-loaded } \mathrm{pH} \text {-sensitive } \\
\text { micelle }\end{array}$ \\
\hline
\end{tabular}

Enzyme-sensitive PTX-loaded matrix MMP-sensitive micelle/Twist-siRNA nanocomplex

MMP-sensitive hybrid liposome containing PTX-loaded pH-sensitive micelle, CSCs inhibitor thioridazine, and the PD-1/ PD-L1 inhibitor HY19991

Legumain-sensitive melittin-modified micelle delivering cabazitaxel

Redox-sensitive

Light-activated NDDSs

Glutathione-sensitive DTX-loaded micelle incorporated into MMP-responsive liposomes

Nanoparticle co-delivering acidity-/ reduction-dual responsive OXA prodrug and reduction-activatable homodimer of NLG919

Liposomal vesicles containing the reactive oxygen species-activatable DOX pro-
Overcoming tumor metastasis as well as drug

Crosslinking DNA and down-regulating the expression of NF-KB simultaneously, inhibit-

Less clearance in blood and effective cellular

tumoral number of CSCs and enhancing antidrug and a PEG-modified photosensitizer pyropheophorbide-a

ICG-templated self-assembly PTX-loaded nanoparticle

Size-switchable shrapnel drug delivery,

Light-inducible nanocargo composed of a reduction-responsive heterodimer of pyropheophorbide-a, an IDO-1 inhibitor and a light-activatable prodrug of OXA The photosensitizer chlorin e6, DTX and Twist siRNA co-loaded nanoparticle for photodynamic/chemo-/gene triple therapy resistance ing tumor growth and metastasis Mitigating $\alpha 4$-integrin/VCAM-1 interaction and effectively accumulating in metastatic sites to inhibit the growth of lung metastases Switchable surface charges, rapid drug release in endosomes/lysosomes, suppressing primary tumor and lung metastasis of breast cancer

Long circulation in blood, effective cancer cellular uptake, superior curative performance on metastatic breast cancer uptake by cancer cells, reducing the intratumor immune responses

Delivering micelles to deep tumor sites, $\mathrm{pH}$ sensitive drug release, and inhibiting the primary tumor growth and the formation of metastasis increasing DTX accumulation in tumors and lungs, realizing satisfactory suppressing effect on the growth and metastasis of breast cancer

Circumventing tumor immunosuppression and exerting encouraging anti-tumor metastasis and recurrence-suppressing effects

Site-specific chemotherapy and photodynamic therapy, improving the outcomes of TNBC treatment

Synergistic effect of photodynamic therapy and immunotherapy with simple production procedure moimmunotherapy, efficient inhibition of breast cancer metastasis and occurrence

Eradicating mesenchymal-like perinecrotic TNBC cells in the deep layer of the tumors, prohibiting the epithelial-mesenchymal transition of perinecrotic TNBC cells in heterogeneous tumor 
Table 2: (continued)

$\begin{aligned} & \text { NDDSs for treat- } \\ & \text { ing metastatic }\end{aligned}$
breast cancer

\begin{tabular}{lll}
\hline Bio-mimetic & Cell membrane- & RBC membrane-coated nanoviscle con- \\
NDDSs & coated NDDSs & $\begin{array}{l}\text { taining methylene blue and cisplatin, } \\
\text { inspired by cytotoxic T cell }\end{array}$
\end{tabular}

Embedding the photosensitizer DiR into the RBC membrane and using it to wrap the PTX-loaded polycaprolactone nanoparticle

Constructing 4T1 cell membrane-coated PTX-loaded nanoparticle

Light-sensitive cancer cell membranescoated DOX-loaded gold nanocage

Monocyte/macrophage membranecoated acid-sensitive liposomes containing the chemotherapeutic drug maytansine derivative

NDDDs loaded in Monocytes loaded with PTX-loaded pHcells

Endogenous nanostructurebased NDDSs

Other formations Local injectable hydrogel sensitive micelles

Monocytes loaded with legumainmotivated nanoparticles delivering maytansine

Bioengineered macrophage-delivering the redox-sensitive prodrug of cytotoxic soravtansine

Ferritin biomimetic nanocage system containing the photothermal therapeutic agent DiR and chemotherapeutic epirubicin

Nanosystem containing IR-780 and Twist siRNA masked by albumin biomimetic nanocorona formed by HSA

Bioinspired lipoprotein nanosystem that respectively loaded the photothermal agent DiR and an anticancer drug mertansine

PEGylated phospholipids membranecoated catalase nanoparticle

Personalized tumor vaccine prepared by incorporating tumor-penetrable peptides into temperature-sensitive hydrogels containing an immunosuppressive regulatory molecule JQ1 and photothermal molecule ICG-carrying tumor cells
Achieving the synergy of photodynamic and

chemotherapy which is traceable by real-time monitoring, suppressing the primary TNBC tumors and inhibiting the formation of lung metastatic nodules

Synergizing photothermal therapy and chemotherapy, inhibiting the lung metastasis of mammary tumor, and serving as in vivo tumor imaging probe

Enriching PTX in primary tumors and metastatic sites through homotypic targeting and inhibiting tumor progression efficiently Combining photothermal therapy and chemotherapy, effectively inhibiting tumor growth and metastasis

Being recruited to tumor sites, efficiently targeting and inhibiting breast cancer lung metastasis

Enhancing the penetration and accumulation of PTX in primary tumors and pulmonary metastatic nodules Significantly improving the tumor-targeting delivery of cytotoxic agents, presenting a promising lung metastasis inhibition ratio Stepwise release of drug-loaded nanovesicles and free drugs in metastases, significantly inhibiting tumor lung metastasis Penetrating into deep tumor tissues and combining photodynamic therapy with chemotherapy to exert sufficient anti-breast cancer lung metastasis effects

Extended blood circulation time and stronger tumor penetration, effectively inhibiting tumor growth and lung metastasis

Clearing up the intra-tumoral delivery obstacles and resulting in efficient inhibition of tumor growth and suppression of lung metastasis of $4 \mathrm{~T} 1$ tumor Tumor oxygenation aided chemophotodynamic therapy inducing significant inhibition of 4T1 tumor growth and striking suppression of lung metastasis

Activating tumor-specific immune memory effects and inhibited the recurrence and metastasis of breast cancer after surgery 
Table 2: (continued)

\begin{tabular}{|c|c|c|c|c|}
\hline $\begin{array}{l}\text { NDDSs for treat- } \\
\text { ing metastatic } \\
\text { breast cancer }\end{array}$ & Property & Design & Key advances & References \\
\hline & \multirow[t]{2}{*}{$\begin{array}{l}\text { Inorganic } \\
\text { nanoparticles }\end{array}$} & $\begin{array}{l}\text { DNA-entangled gold nanorods loaded } \\
\text { with DOX }\end{array}$ & $\begin{array}{l}\text { Promoting tumor cell apoptosis and shrinking } \\
\text { the blood vessels at the tumor sites, inhibit- } \\
\text { ing the growth of primary tumors and sup- } \\
\text { pressing lung metastasis }\end{array}$ & [80] \\
\hline & & $\begin{array}{l}\text { Incorporating DOX into iron-containing } \\
\text { mesoporous silicon nanoparticles via } \\
\text { metal-ligand coordination bonding }\end{array}$ & $\begin{array}{l}\text { Achieving the real time monitoring of anti- } \\
\text { cancer drugs release by T1-weighted mag- } \\
\text { netic resonance imaging, enhancing anti- } \\
\text { tumor efficacy and inhibiting the lung } \\
\text { metastasis of the tumor }\end{array}$ & [81] \\
\hline & Liposomes & $\begin{array}{l}\text { Phospholipid-mimic OXA prodrug } \\
\text { liposome }\end{array}$ & $\begin{array}{l}\text { High drug loading capacity, longer blood cir- } \\
\text { culation time, increased tumor accumulation } \\
\text { and significantly inhibiting the progression of } \\
\text { TNBC }\end{array}$ & [23] \\
\hline
\end{tabular}

CSC: cancer stem cells; DOX: doxorubicin; HSA: human serum albumin; ICG: indocyanine green; IDO: indoleamine 2,3-dioxygenase; MMP: matrix metalloproteinase; NF-KB: nuclear factor kappa B; OXA: oxaliplatin; PD-1: programmed cell death protein one; PD-L1: programmed death-ligand one; PEG: polyethylene glycol; PTX: paclitaxel; RBC: red blood cell; TNBC: triple negative breast cancer; VCAM-1: vascular cell adhesion molecule-1.

According to the findings above, a series of NDDSs based on different materials, including stimuli-responsive polymers, cell-derived and other endogenous components, lipids and inorganic nanoparticles, have been constructed by our team (Figure 1 and Table 2). The details about their formulations and the effects on suppressing metastatic breast cancer will be introduced below.

\section{Stimuli-responsive polymeric NDDSs}

Polymer-based nanocarriers with versatile structures enrich the toolbox for designing multi-functional NDDSs. Specific ligands can be conjugated on polymer skeletons for targeting delivery. In recent years, various medicinal functional biomaterials containing modules sensitive to specific stimuli in the tumor microenvironment have been synthesized, and drug delivery vehicles with the capability of intelligent and controllable drug release have been constructed, which are encouraging for overcoming physiological barriers and delivering drugs into solid tumors or metastasis sites. In addition, a series of light-responsive NDDSs applied for photothermal or photodynamic therapy which inhibited the growth of primary tumors and lung metastasis were also designed and prepared.

\section{pH-responsive NDDSs}

Protonable polymers are the most frequently applied materials to achieve $\mathrm{pH}$-sensitive drug release, which is timesaving and leads to endosome/lysosome disruption to accelerate drug release in cytosol. For the core-shell structure nanocarriers consisting of $\mathrm{pH}$-sensitive amphiphilic polymers, drugs could be stably encapsulated under physiological conditions. After entered tumor cells, lower $\mathrm{pH}$ in endosomes/lysosomes ( $\mathrm{pH}$ 4.5-5.5) caused protonation of the core and dissociation of the carrier, leading to timely release of cargos inside.

The acid-sensitive amphiphilic polymer, BD-PEI, can self-assemble to form stable micelles in water [44]. The $\mathrm{N}, \mathrm{N}$-diisopropylethylenediamine (DPA) group on BD-PEI would undergo protonation at $\mathrm{pH}$ values less than 6.3, resulting in the transformation of the micelle core from hydrophobic to hydrophilic. Moreover, DPA possesses the membrane-disrupting function, which would benefit the endo/lysosomal escape of the cargo [82]. The downregulation of AKT, a linchpin of the phosphatidylinositol 3-kinase (PI3K)/AKT/mammalian rapamycin (mTOR) pathway, often restricts the development of drug resistance and metastasis of cancer [83, 84]. Yin et al. [65] utilized BD-PEI to construct the pH-sensitive PTX-loaded micelle/ siAkt nano-complex (PMA) for interfering Akt gene expression. PMA with the mean particle sizes of $50-80 \mathrm{~nm}$ 
kept intact in blood circulation and disintegrated in the acidic environment of lysosomes. After the lysosomal membranes were damaged by PMA, PTX and siAkt entered the cytoplasm to play the role of anti-tumor growth and increasing the sensitivity of cells to chemotherapy. The tumor volumes in the 4T1 breast cancer mice model treated with PMA decreased more significantly than other groups, with less lung metastatic foci observed, indicating the advantages of combined treatment strategies in overcoming tumor metastasis and drug resistance [65]. Subsequently, another BD-derived block polymer, BD-PEG, was synthesized, based on which the DTX- and silibinin (SIL)- codelivering micelle (DSM) was developed [85]. SIL was found to be potential in inhibiting tumor cell migration and invasion via regulating EMT [86]. DSM was stable in blood circulation and could be efficiently taken up by tumor cells. Then DSM rapidly dissociated and released DTX and SIL under low $\mathrm{pH}$ conditions in tumor cell endosomes. The codelivery of DTX and SIL by DSM showed significant inhibition effect on breast cancer lung metastasis [85]. Fabricated through the thin film hydration method, micelles based on BD derivatives provided a convenient and efficient platform to improve the curative performance of chemotherapeutic agents and anti-metastatic agents.

Blocking Twist and nuclear factor kappa B (NF- $\mathrm{kB})$ pathways is anticipated to inhibiting cell invasion and metastasis efficiently. Self-assembled three-layer micelleplexes loaded with a cisplatin prodrug and the siRNA targeting the NF- $k B$ pathway (siRNA-p65) were prepared by Yu et al. [66],using a pH-sensitive and amphiphilic triblock copolymer PEG-block-poly(aminolated glycidyl methacrylate)-block-poly[2-(diisopropyl amino) ethyl methacrylate] (PEG- $b$-PAGA- $b$-PDPA). The cisplatin prodrug was encapsulated in the hydrophobic PDPA core and siRNA was condensed by the positively charged PAGA. After micelleplexes were endocytosed into tumor cells, the protonation of PDPA resulted in the disintegration of micelleplexes in lysosomes. The cisplatin prodrug and siRNA were released into the cytoplasm. Cisplatin was activated by the reduced glutathione, and siRNA-p65 down-regulated the expression of NF- $\mathrm{kB}$, inhibiting tumor growth and metastasis. This drug delivery system inhibited tumor metastasis by co-delivering the anti-NF- $\kappa B$ siRNA with the prodrug of chemotherapy agent and controlling the release and activation of them, achieving combinational therapeutic outcome [66].

$\beta$-cyclodextrin $(\beta-C D)$ and amantadine have exhibited high affinity for constructing host-guest nanocomplexes, and these two kinds of molecules can be conjugated with functional groups to develop demanded drug delivery systems [87]. Dan et al. [67] synthesized the acid-sensitive host polymer, DPA-modified $\beta$-CD ( $\beta C D$-DPA), and the guest polymer, amantadine-modified PEG. Making use of the host-guest interaction of $\beta-C D$ and hydrophobic amantadine, they constructed a $\mathrm{pH}$-responsive host-guest nanosystem (PHN) containing succinobucol. After entering the lysosomes in tumor cells, DPA protonation occurred immediately and the nanosystem quickly released payloads under acidic conditions, which significantly inhibited tumor cell invasion/migration and interaction with macrophages probably through mitigating VCAM-1 expression and $\alpha 4$-integrin/VCAM-1 interactions. PHN also effectively accumulated in metastatic sites after intravenous administration and inhibited the growth of lung metastases [67].

The precise and intelligent drug delivery systems with the "core-shell-corona" structure can display a selfadaptive ability in vivo. In the weakly acidic tumor microenvironment ( $\mathrm{pH} \sim 6.8$ ), the corona, i.e. the outermost layer, conjugated on nanovehicles via pH-labile covalent bonds was sheared off, leading to the increase of the surface charge to a more positive level, which facilitate the cellular uptake of nanoparticles. Combining with the acid-triggered drug release strategy, the dual $\mathrm{pH}$ responsive NDDSs efficiently improve the accumulation of therapeutic agents in both primary solid tumor and metastatic foci. Tang et al. [24] synthesized acid-sensitive amphiphilic polymers PEG-block-poly[(1,4-butanediol)diacrylate- $b$-5-amino-1-pentanol] (PEG-PDHA) and 2,3-dimethylmaleic anhydride conjugated with PEI-blockPDHA (DMA-PEI-PDHA). Two polymers self-assembled in aqueous solution to construct dual pH-sensitive PTX-loaded micelles (DPM). In the blood circulation, DPM remained stable with negative surface potential. Once reaching the tumor tissue, DMA was sheared off in response to the special $\mathrm{pH}$ and the surface charge of DPM was reversed to positive, thus promoting the specific internalization by cells in primary tumors and metastatic foci. In the lysosomes of tumor cells, the PDHA core was further protonated quickly. Disintegration of nanostructures facilitated the escape of PTX from endosomes/ lysosomes, achieving rapid and sufficient intracellular drug release. In the 4T1 pulmonary metastatic mice model, anti-tumor and anti-metastatic efficacy of DPM were verified to be superior to the micelles which were non-sensitive to the acidic $\mathrm{pH}$ [24].

The DMA-based charge-switching strategy was applied in another anti-metastasis study. The active vitamin D metabolite, 1,25-dihydroxyvitamin D3 (calcitriol, CTL), has exhibited activities to inhibit the pro-tumoral signaling pathways in breast cancer cells [88]. With the purpose of precise tumor-targeting delivery of CTL and PTX with 
ameliorated side effects and improved efficacy, the PTX/ CTL-co-loaded pH-sensitive micelle (PCDM) was constructed by Zheng et al. [68] At neutral pH, PCDM showed negative zeta potential and escape from the mononuclearphagocyte system, while the abscission of DMA and surface charge reversal would be induced by acidity in tumor tissue. The acid-labile core containing DPA groups ensured rapid release of PTX and CTL from endosomes/lysosomes, thus significantly suppressed primary tumor growth and lung metastasis of breast cancer. Moreover, enhanced expression of $\mathrm{C}-\mathrm{C}$ motif chemokine ligand 2 (CCL2) and expansion of $\mathrm{Ly} 6 \mathrm{C}^{+}$monocytes in lungs induced by PTX might promote metastasis, but this effect could be counteracted by CTL. The intra-tumoral concentrations of PTX and CTL of the PCDM group reached 46.68 and 26.50 times of those of the free drug group, respectively, demonstrating that PCDM increased the intra-tumoral distribution of its cargo. In addition, administration of PCDM avoided the heart damage and bone marrow suppression caused by free PTX and CTL, which indicated that PCDM might be a promising alternative of free drugs for clinical use with less toxicity and good compatibility [68].

\section{Enzyme-sensitive NDDSs}

Altered expression of certain enzymes is another commonly used endogenous stimulus for the design and construction of programmed nanocarriers. A series of NDDSs employing enzyme-/pH- dual sensitive drug release strategies have been reported. In the tumor tissues with metastatic potential, the MMP family is one kind of the most studied overexpressed enzymes [37]. MMP-2/9-cleavable peptide with the sequence of Pro-Leu-Gly-Leu-Ala-Gly (PLG) was exploited to synthesize MMP-sensitive polymers and develop enzyme sensitive systems. Tang et al. [25] synthesized an amphiphilic block copolymer PEI-PDHA and an enzyme-sensitive polymer PEG-p-PDHA, and constructed micelles for the combined delivery of PTX and anti-Twist-siRNA. The PEG corona helped with the retention of the PTX-loaded micelle/TwistsiRNA nanocomplex in blood circulation. After reaching the tumor or the metastatic site, PEG was removed under the action of MMP and the positive charge was exposed to induce effective tumor cell uptake. After entering the lysosomes, PDHA was protonated, which facilitated the lysosomal escape of PTX and siRNA, inducing cancer cell apoptosis and down-regulating the expression of Twist for metastasis suppression. This dual-sensitive chemotherapeutic agent/ siRNA co-delivery system exhibited superior curative performance on metastatic breast cancer compared with its nonMMP-sensitive counterpart [25].
Lang et al. [56] reported a cocktail delivery strategy, integrating chemotherapy against solid tumor cells and cancer stem cells (CSCs) and immune checkpoint blockade therapy into one spatio-temporally controlled NDDS (Figure 2A). They synthesized the pH-responsive BD-PEG (PDB) and conjugated PEG to vitamin E succinate through PLG (PPV). PDB was used to construct PTX-loaded micelles, which was encapsulated in hybrid liposome consist of the cholesterol, soybean phosphatidylcholine and PPV together with the anti-CSC agent thioridazine (THZ) and the small molecular programmed cell death-1/programmed cell death-ligand 1(PD-1/PD-L1) inhibitor HY19991. The relatively big particle size of the micelle@liposome system led to less clearance in vivo. In tumor tissues with overexpressed MMP, the liposome dissociated and released the micelles, THZ and HY19991. The small-sized PTX-loaded micelles would be more easily internalized by tumor cells and CSCs, then killed them. The dual responsive NDDS with MMP-sensitivity and acid-sensitivity could effectively reduce the number of CSCs and Tregs, as well as increased the number of $\mathrm{CD}^{+} \mathrm{T}$ cells infiltration in tumor tissues (Figure 2B-D). Effector memory $\mathrm{T}$ cells in blood, spleen, and bone marrow were increased. The cocktail delivery strategy presented efficient inhibition effects on lung metastasis of breast cancer, providing new ideas for treating breast cancer metastasis and preventing tumor recurrence (Figure 2E, F) [56].

Legumain protease is highly expressed in the tumor microenvironment, but barely detected in normal tissues. He et al. [69] constructed a legumain-sensitive melittin (legM)-modified micelle delivering the microtubule inhibitor cabazitaxel (TCM-legM) (Figure 3A). The micelles were intact in the blood with the average diameter of $45.9 \mathrm{~nm}$. When TCM-legM reached the tumor microenvironment highly expressing legumain, a substrate peptide in legM would be recognized and cleaved by legumain and legM could be transformed into active melittin (Figure 3B). The restored melittin exerted the effect of penetrating the membrane and delivering micelles to deep tumor sites (Figure 3C, D). Endosomal/lysosomal acidic environments induced the disintegration of micelles to release the encapsulated cabazitaxel, inhibiting the growth of primary tumors and the formation of metastasis, confirming the superiority of the legumain-/pH- dual responsive delivery strategy (Figure 3E, F) [69].

\section{Redox-sensitive NDDSs}

Reduction-sensitivity has been widely utilized for efficient and selective drug delivery to malignant cancer due to high 
A

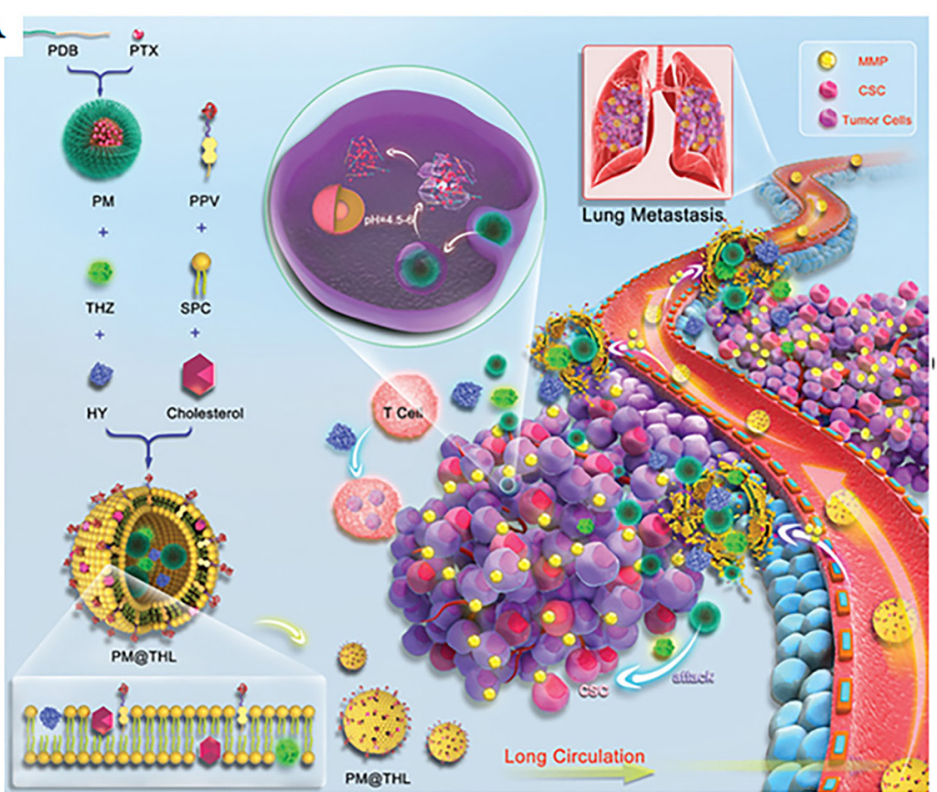

D

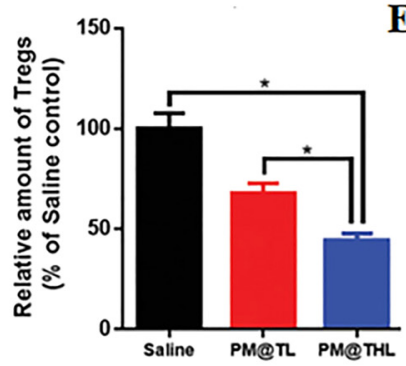

$\mathbf{E}$

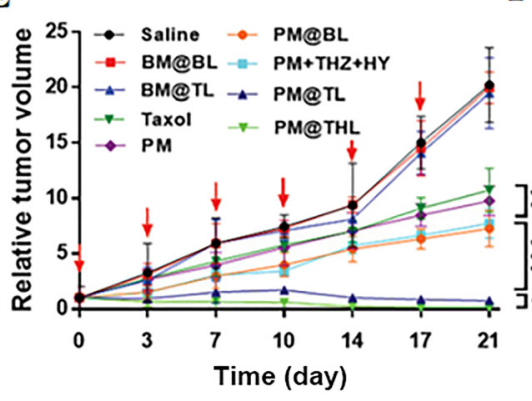

B
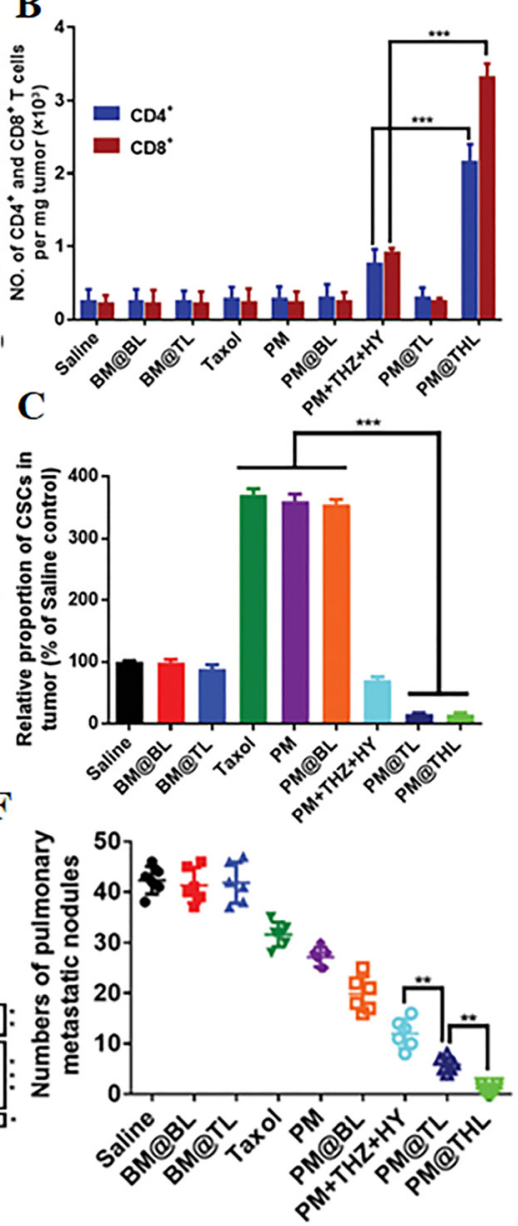

Figure 2: The cocktail strategy based on the spatio-temporally controlled nano device improved breast cancer therapy. (A) Cocktail therapy of metastatic breast cancer by PM@THL with the dual sensitive double-layer structure. (B) The numbers of CD4 ${ }^{+}$or CD8 ${ }^{+}$T cells in tumors. (C) The CSC proportion within tumors. (D) the relative amount of Tregs $\left(\mathrm{CD}^{+} \mathrm{CD} 25^{+}\right.$Foxp $\left.3^{+}\right)$in tumors. (E) the profiles of tumor volumes variation. (F) Numbers of the pulmonary metastatic nodules. Data are shown as mean $\pm \operatorname{SD}(n=6) .{ }^{*} p<0.05,{ }^{* *} p<0.01,{ }^{* \star *} p<0.001$. BL: blank micelle-loaded hybrid liposome; BM: blank micelle, CSC: cancer stem cells; HY: HY19991; MMP: Matrix metalloproteinases; PDB: Polyethylene glycol-blockpoly[(1,4-butanediol)-diacrylate- $\beta$ - $N, N$-diisop ropylethylenediamine; PM: PTX-loaded PDB micelle; PPV: Methoxy polyethylene glycol-peptidevitamin E succinate; PTX: paclitaxel, SD: standard deviation;SPC: Soybean phosphatidylcholine; THL: THZ/HY-loaded hybrid liposome; THZ: thioridazine; TL: THZ-loaded hybrid liposome. Reproduced with permission [56]. Copyright 2018, Wiley-VCH.

intracellular reductive glutathione concentration in tumor cells [89]. Several NDDSs combining the redox-sensitive strategy with other strategies to elevate the drug concentration in tumor cells and improve the outcomes of antimetastatic treatment have been reported. Xiao et al. [26] synthesized a reduction-sensitive amphiphilic block polymer Tween 85-disulfide bond-polyethyleneimine 2K (TSP), which could self-assemble in water to form micelles with positive charges for carrying the shRNA silencing NF- $\mathrm{kB}$ (shp65). Tween 85 was employed in this nanosystem to ensure the stability in blood circulation and increase uptake by tumor cells via interacting with the low-density lipoprotein receptor. After disintegration in tumor cells, the micelle/shp65 nanocomplex released siRNA rapidly, which effectively inhibited proliferation and invasion of tumor cells by down-regulating the NF-kB signal pathway, resulting in suppressed growth and metastasis of MDA-MB435 tumors. This reduction-sensitive drug delivery system provided promising approach to improving RNA interference therapy of metastatic breast cancer [26].

$\mathrm{Xu}$ et al. [70] synthesized reduction-sensitive amphiphilic block copolymer glycol-disulfide bond-vitamin E succinate (PSV) to prepare a glutathione-sensitive DTX-loaded nanomicellar system (DPM) (mean particle size $<20 \mathrm{~nm}$ ). Subsequently, DPM was incorporated into MMPs-responsive liposomes with the particle sizes of about $100 \mathrm{~nm}$ based on PPV. This drug delivery system firstly responded to the up-regulated extracellular MMPs 
A

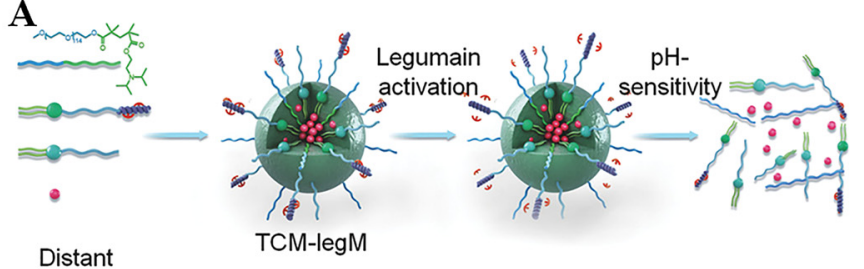

Distant metastases

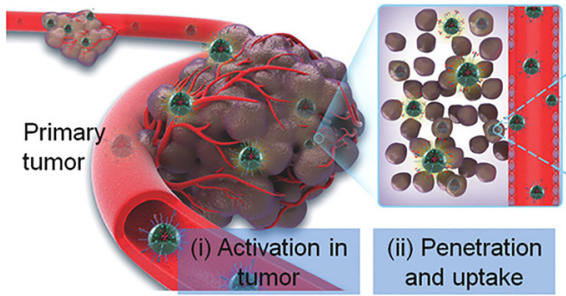

B

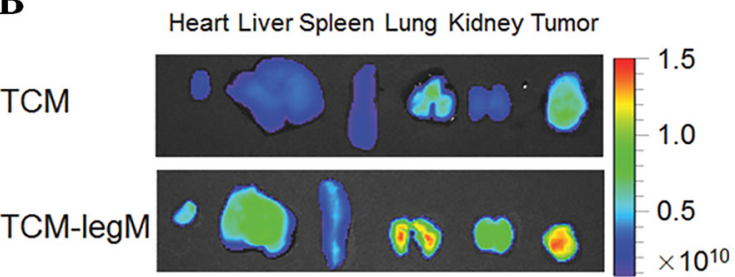

C
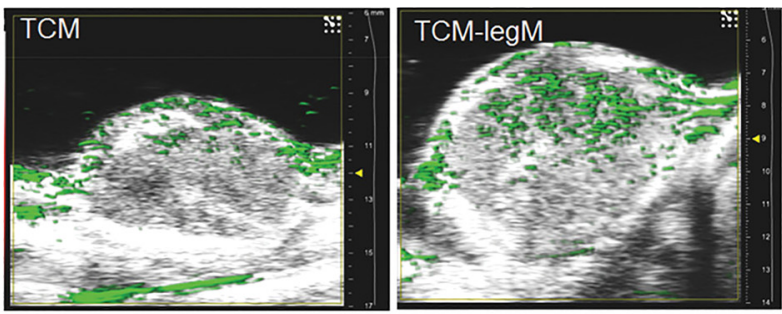
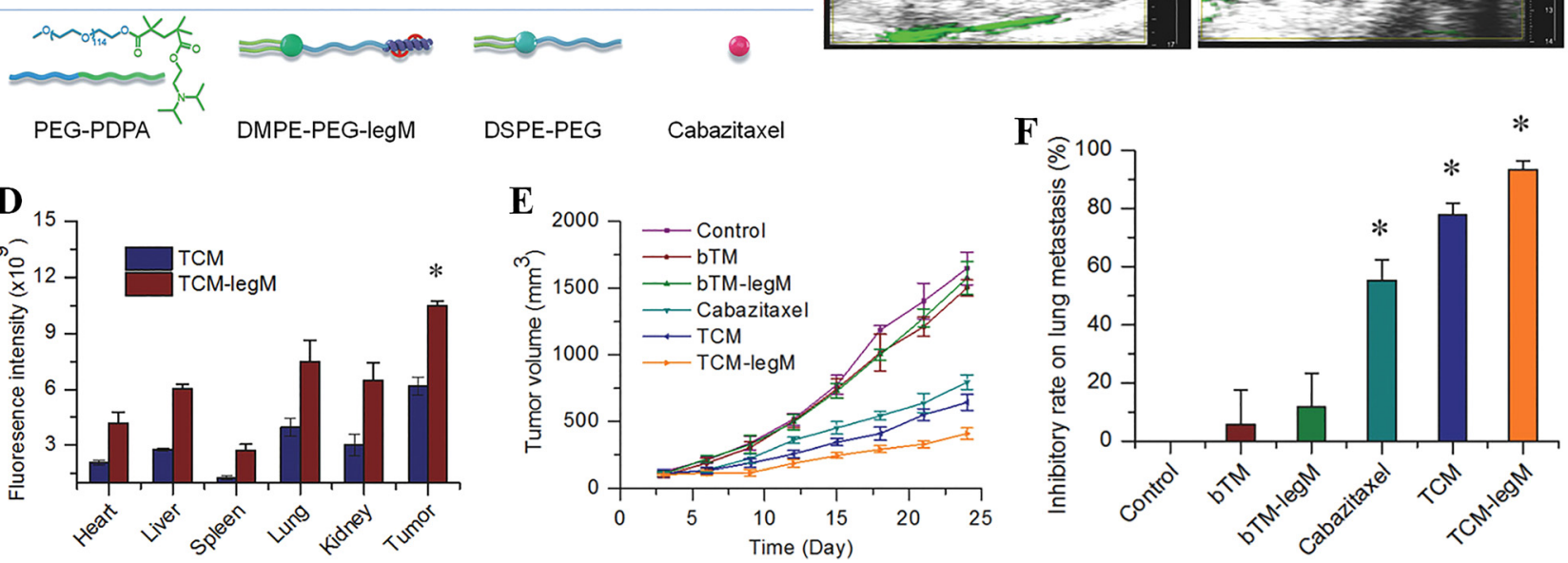

Figure 3: Rational design of tumor microenvironment-activated micelles for programed targeting of breast cancer metastasis. (A) Schematic illustration of TCM-legM for programed targeting of breast cancer metastasis, which can be divided into three steps: (1) specific activation by the highly expressed legumain protease in tumor microenvironments; (2) promoting the deep tumor penetration and efficient internalization by cancer cells. (3) motivating the $\mathrm{pH}$-responsive drug release in intracellular acidic environments to exert the antimetastatic effects. (B) The distribution of TCM-legM and TCM in major organs at $4 \mathrm{~h}$ post injection. (C) The deep penetration of TCM-legM in primary tumor determined by photoacoustic imaging. (D) The quantified distribution of TCM-legM and TCM in major organs. ${ }^{*} \mathrm{p}<0.05$ (the TCM group vs. the TCM-legM group). (E) The tumor growth profiles of each group. (F) The inhibition rate of various formulations on lung metastasis. ${ }^{*}<<0.05$ vs. the cabazitaxel group and the TCM group. DMPE: 1,2-Dimyristoyl-sn-glycero-3-phosphoethanolamine; DSPE: 1,2-Distearoyl-sn-glycero-

3-phosphoethanolamine; TCM: Tumor microenvironment-activated cabazitaxel micelles; TM: blank micelles; PEG: poly(ethylene glycol); PDPA: Poly (2-diisopropyl methacrylate). Reproduced with permission [69]. Copyright 2017, Wiley-VCH.

concentration in tumors, followed by further drug release under the intracellular reductive environment. This double-responsive shrapnel nanosized drug delivery system with high encapsulation efficiency (99.02\%) and obvious burst release (>90\%) effectively increased DTX accumulation in tumors and lungs after intravenous injection and realized satisfactory effect to suppress the growth and metastasis of breast cancer [70].

Besides synthesizing bioreducible polymers as drug carriers, reduction-responsive prodrugs can be a choice to specifically activate drugs inside tumor cells. Immunotherapy, which eliminates cancer cells via the immune responses by reactivating dendritic cells and cytotoxic $\mathrm{T}$ cells, is promising for treating metastasis cancer.
However, the low tumor immunogenicity and immunosuppressive tumor microenvironment lead to the low response rate. Chemotherapeutic agents such as oxaliplatin (OXA) can induce immunogenic cell death and increase tumor immunogenicity, which facilitates tumor antigen presenting and intra-tumoral recruitment of cytotoxic $\mathrm{T}$ cells [90]. The combination of chemotherapeutic agents with the immune modulators of the tumor microenvironment can be achieved by being co-delivered by NDDSs to enhance the anti-tumor responses. Feng et al. [71] designed and constructed a smart binary cooperative prodrug nanoparticle (BCPN) to achieve dual activatable and highefficient tumor/metastasis inhibition by combining tumor immunotherapy and chemotherapy. BCPN co-delivered the 
acidity- and reduction-dual responsive OXA prodrug and the reduction-activatable homodimer of the indoleamine 2,3-dioxygenase 1 (IDO-1) inhibitor NLG919 to tumor tissues. Cleavage of the PEG corona in acidic tumor microenvironment switched surface charges to promote tumor penetration and cellular uptake. In the reduction environment of tumor cells, the prodrugs were reduced and activated to release active OXA and the NLG919 monomer. OXA could induce immunologic cell death of tumor cells and improve the tumor immunogenicity, thus stimulated the body's adaptive anti-tumor immune responses, indicated by improved infiltration of cytotoxic T cells in tumor tissues. NLG919 effectively inhibited the IDO-1 enzyme activity to circumvent the immunosuppression of the tumor microenvironment. BCPN exerted encouraging antitumor metastasis and recurrence effects. This work had noticeable value for the development of safe and efficient tumor immunotherapy. Satisfactory drug encapsulation efficacy, adjustable drug loading ratios and highly controllable formulation procedures facilitated the scaleup and clinical application of BCPN [71].

\section{Light-activated NDDSs}

As an accessible exogenous stimulus, NIR laser irradiation has attracted increasing attention due to high tissue penetration and noninvasive procedure. The first NIR window (NIR-I) from 700 to $1000 \mathrm{~nm}$ has been extensively investigated for deep-tissue imaging due to the reduced blood absorption in this region. The photosensitizers include several metallic substances, inorganic agents (e.g., carbon nanotubes and quantum dots), and organic small molecules [e.g., indocyanine green (ICG) and methylene blue] [91]. Compared to NIR-I, NIR-II window (1,000-1,400 nm) offers numerous merits, including deeper light transmission inside tissue, longer wavelength excitation, higher spatial resolution, minimal autofluorescence, and reduced tissue scattering, showing tremendous potential for imaging and photothermal therapy of tumors [92]. Encouragingly, increasing studies in recent years demonstrated a good application prospect of nanotechnology-based cancer theranostic with the assistance of NIR-II.

Photodynamic therapy (PDT) is one of the most exploited light-activated therapy of tumor. Assisted by photosensitizers, laser light and oxygen, PDT induces cytotoxic ROS generation to achieve therapeutic effects such as antitumor immune response initiation and ECM degradation. In order to improve the efficacy of PDT, several nanotechnology-based drug delivery systems for combining PDT with immunotherapy and/or chemotherapy has been reported.
Zhou et al. [72] constructed liposomal DOX prodrug vesicles containing the ROS-activatable DOX prodrug and a PEG-modified photosensitizer pyropheophorbide-a ( $\mathrm{PPa})$ (RADV) (Figure 4A, B). Both fluorescence and photoacoustic tumor imaging guided the laser to irradiate on the exact location of tumors and generate ROS to activate the DOX prodrug for site-specific chemotherapy and PDT. Moreover, ultrafast release of DOX prodrug was realized by the increase of lipid membrane permeability induced by ROS-mediated oxidation of the unsaturated 1,2-dioleoyl-snglycero-3-phosphocholine (DOPC), ensuring sufficient drug exposure to tumor cells (Figure 4C). This ROS-triggered theranostic system significantly inhibited the tumor growth and distant metastasis of triple negative breast cancer (TNBC) (Figure 4D, E), with negligible toxic effects on major organs. Therefore, this study provided an alternative option for improving the outcomes of PDT and chemotherapy for treating metastatic TNBC [72].

Low immunogenicity and immunosuppressive tumor microenvironment diminish the efficacy of PDT immune checkpoint block therapy. The co-delivery of immune checkpoint inhibitors and photosensitizers via simple procedures without excipients was firstly reported by Feng et al. [73] They found a 2-in-1 nanomedicine based on the ICG-templated self-assembly strategy, realizing nearly $100 \%$ loading of various small molecular drugs without any excipient. As a representative, ICG-templated self-assembled PTX nanoparticles (ISPN) were studied. In this research, the cytotoxic $\mathrm{T}$ cells to regular $\mathrm{T}$ cells (Tregs) ratio and the intra-tumoral content of inflammatory cytokines [interferon (IFN)- $y$, tumour necrosis factor (TNF)- $\alpha$, and interleukin (IL)-6)] in the 4T1 tumor mice model were significantly enhanced by IPSN in combination with the anti-PD-L1 antibody. Based on this promising nanodrug produced by a simple procedure, the synergistic effect of PDT and immunotherapy exerted encouraging performance in tumor suppression, metastasis inhibition, and recurrence prevention [73].

By incorporating photosensitizers, chemotherapeutic agents, and inhibitors of tumor immunosuppression into nanomedicines simultaneously, the combination of PDT and chemo-immunotherapy can be carried out as a promising choice for treating cancer. Feng et al. [62] constructed a light-inducible nanocargo (LINC) composed of a reduction-responsive heterodimer of photosensitizer $\mathrm{PPa}$, IDO-1 inhibitor NLG919, and a light-activatable prodrug of OXA. After intravenous injection, LINC passively accumulated at the tumor site and generated a fluorescence signal. The first wave of NIR laser irradiation guided by the fluorescence signal led to the generation of ROS and cleavage of PEG corona, which ensured the deep penetration of 
A

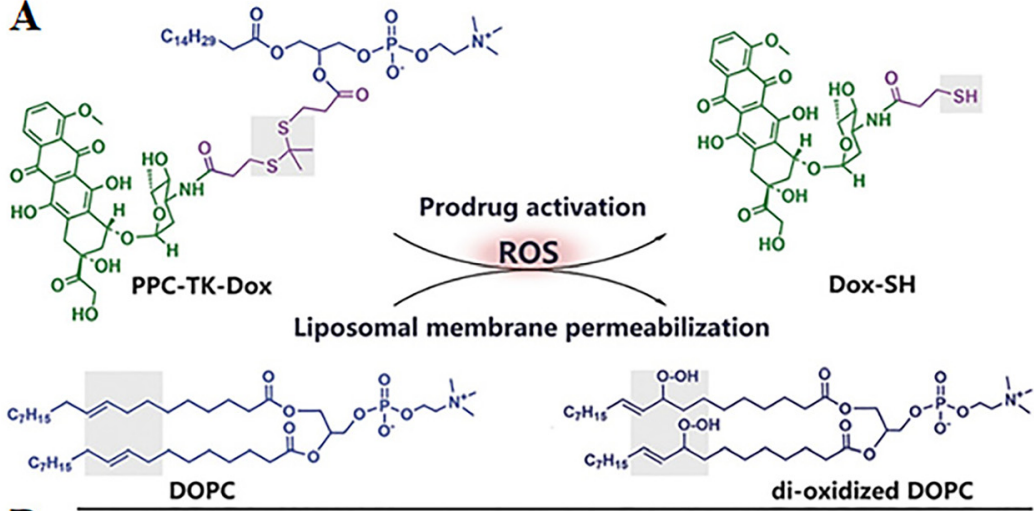

B

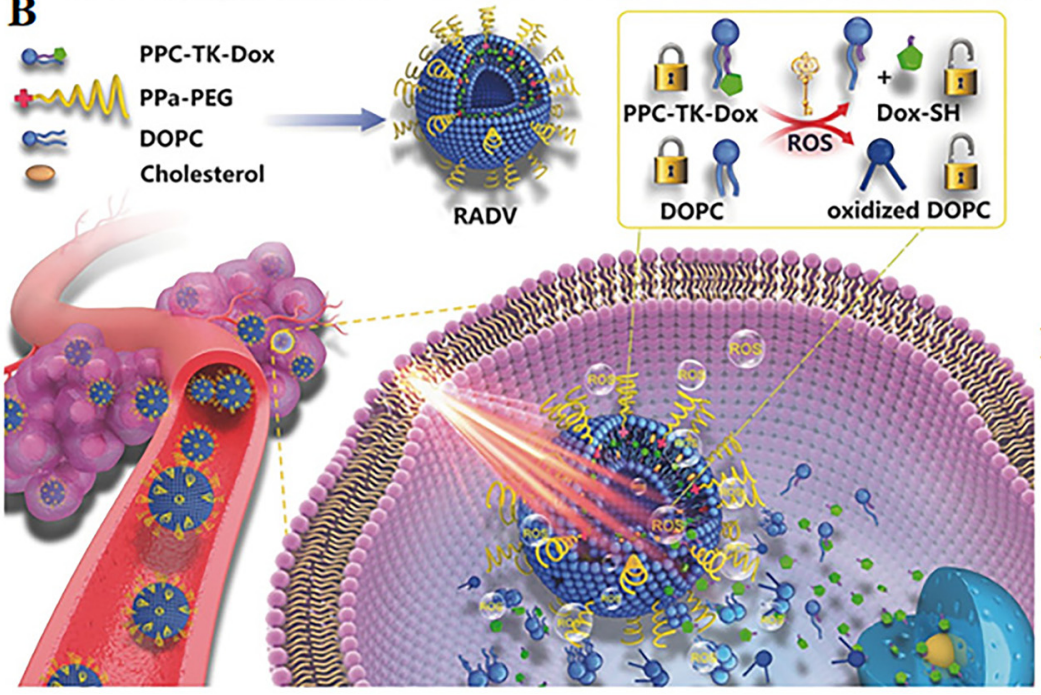

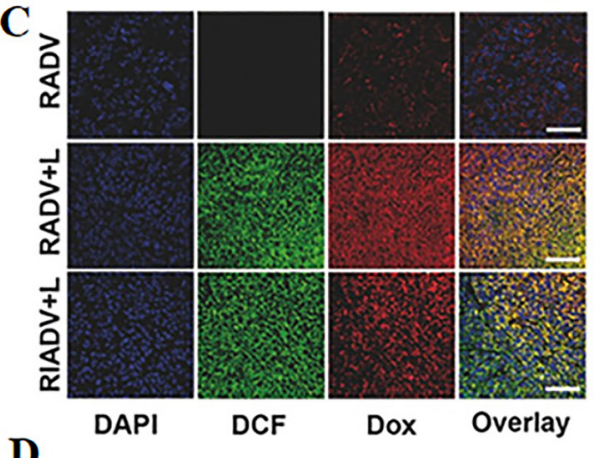

D
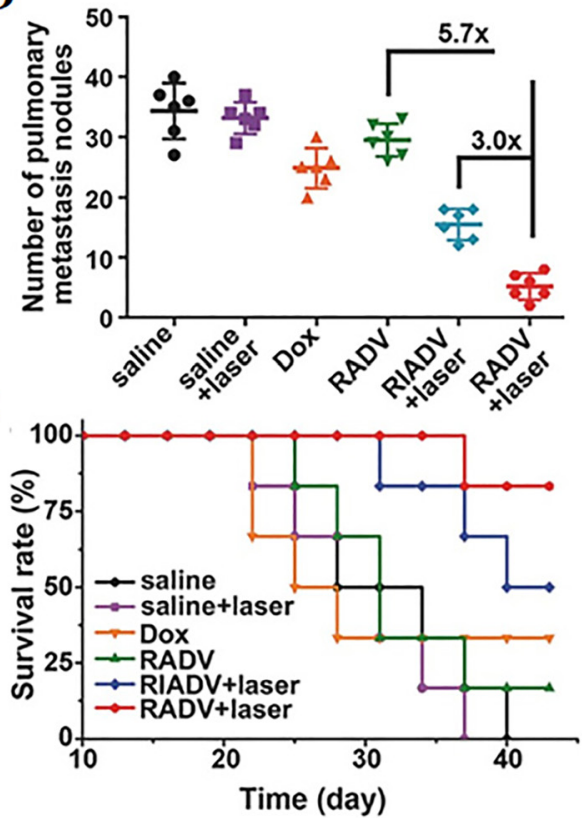

Figure 4: Theranostic prodrug vesicles with ROS-triggered ultrafast drug release for local-regional therapy of metastatic triple-negative breast cancer. (A) Proposed process of ROS-mediated activation of the phospholipid-mimicking DOX prodrug and oxidization of unsaturated DOPC. (B) Schematic illustration of the ROS-activatable DOX prodrug vesicle as a robust nanoplatform for imaging-guided ultrafast drug release and local therapy of TNBC. (C) Confocal laser scanning microscopic (CLSM) examination of laser-triggered ROS generation (scale bars=50 $\mu \mathrm{m})$. (D) The anti-metastasis efficacy of RADV-mediated combination therapy examined by counting pulmonary metastasis nodules. (E) The survival rate of mice received different treatments. DAPI: 2-(4-Amidinophenyl)-6-indolecarbamidine dihydrochloride; DCF: 2,7-Dichlorodihydro fluorescein; DOPC: 1,2-Dioleoyl-sn-glycero-3-phosphocholine; Dox: Doxorubicin; PEG: Poly (ethylene glycol); PPC: 1-Palmitoyl-2-hydroxysnglycero3-phosphocholine; RADV: Reactive oxygen species-activatable doxorubicin prodrug vesicle; ROS: Reactive oxygen species; TK: thioketal; TNBC: Triple negative breast cancer. Reproduced with permission [72]. Copyright 2017, Wiley-VCH.

LINC. Under the second wave of NIR laser irradiation, OXA was completely released and stimulated the immune response with the assistance of IDO inhibition. According to the researchers, intra-tumoral infiltration of the $\mathrm{CD} 8^{+}$ $\mathrm{T}$ cells, the $\mathrm{CD}^{+} \mathrm{T}$ cells to Tregs ratio, the number of memory T cells in spleens, the IFN-y and TNF- $\alpha$ levels in the sera of metastatic 4T1 tumor-bearing mice were significantly enhanced by LINC, which led to the inhibition of breast cancer metastasis and occurrence [62].

The activity-loss of PDT along the light path is a challenge for eradicating mesenchymal-like perinecrotic TNBC cells in the deep layer of the tumors, which contributes to the recurrence and metastasis of the disease. Meng et al. [27] constructed a nanoparticle containing photosensitizer chlorin e6 (Ce6), DTX and anti-Twist siRNA (CDTN) for the photodynamic/chemo-/gene triple therapy of heterogeneous TNBC. The nanoparticle mainly killed rapidly proliferating tumor cells through photodynamic action on the tumor surface with sufficient light illumination. In the deep layer of the tumor lacking effective light, CDTN destroyed the epithelial mesenchymal tumor cells by promoting the escape of chemotherapeutic agents and siRNA from the lysosome, and finally effectively inhibited the growth of tumor in situ and tumor metastasis. This work revealed 
the importance of prohibiting the EMT of perinecrotic TNBC cells in the treatment of heterogeneous tumors, providing a novel platform for the treatment of TNBC [27].

Feng et al. [93] synthesized acid-sensitive Ce6 photosensitizer derivatives (AC) and reduction-sensitive hydrophobic OXA prodrug, hexadecyl-OXA-trimethyleneamine (HOT), and combined them with iRGD modified DSPE-PEG to form prodrug nanoparticles. The nanoparticles circulated in the blood for a long time. Through the recognition of Nrp-1 receptors by CRGDK/R, which was formed by the binding of $\mathrm{iRGD}$ on integrins, the prodrug nanoparticles achieved effective tumor penetration and accumulation. Acid triggered-fluorescence signal generated by AC in tumor sites, acting for cancer imaging, precisely guided photodynamic action. Under the action of infrared light, PDT promoted the lysosomal escape of OXA prodrugs which selectively targeted the mitochondria via electrostatic interactions, cooperating with PDT to exert an antitumor metastasis effect. This versatile prodrug nanosystem presented an option for combinational tumor imaging and organelle-specific therapy [93].

As a promising and noninvasive treatment for cancer, photothermal therapy (PTT) employs photoabsorbers to induce hyperthermia under NIR laser irradiation for thermal ablation of cancer cells [94]. Compared with inorganic photothermal nano agents, NIR fluorescence dyes and their nanoassemblies overcome the disadvantages of long-term toxicity to some extent, thus have better potential to be developed as PTT agents [95]. Several studies on NDDSs based on NIR fluorescence dyes and their nano-assemblies for inhibiting metastasis of breast cancer have been established.

IR-780 has strong light-absorbing capacity, but poor solubility restricts its heat-generating efficacy for PTT application. To circumvent the drawback of IR-780, He et al. [96] constructed the IR-780-loaded DSPE-PEG2000 polymeric micelles (IPMs) for PTT of breast cancer lymphatic metastasis. The micelles were stable with the particle size of $25.6 \pm 4.0 \mathrm{~nm}$ under physiological conditions and efficiently targeted to primary tumor and metastatic lymph node sites. Under laser irradiation, the micelles exhibited significant heat production capacity. At the same time, micelles could accumulate and penetrate in tumors and metastatic lymph nodes. As a result, IPMs significantly inhibited tumor growth and lymphatic metastasis. This micelle provided a new idea for lymphtargeting PTT of metastatic breast cancer [96].

Similarly, in order to achieve a deep tumor-penetrating effect to enhance the PTT efficacy of 1,1-dioctadecyl-3,3,3,3tetramethylindotricarbocyanine iodide (DiR), He et al. [97] encapsulated DiR in PEG-b-PDPA nanoparticles with the mean diameter within $20-30 \mathrm{~nm}$, which was regarded as the size with optimal tumor-penetration capacity. Due to the high local concentration of DiR in the hydrophobic core, its photothermal effect was greatly enhanced compared with free DiR. The assembled nano-drug effectively accumulated in the tumor sites and generated local high temperatures under infrared light irradiation of $808 \mathrm{~nm}$, resulting in the significant inhibition of cell proliferation and migration activity. The local high temperatures promoted the penetration of nanoparticles into the tumor sites. At the same time, the photothermal actions completely killed the tumor cells after a single treatment, inducing the tumor regression and preventing the occurrence of lung metastasis [97].

\section{Biomimetic NDDSs}

During the process of primary tumor growth and metastatic sites, the interaction between cancer cells and a variety of accessory cells including mesenchymal stem cells (MSCs), monocytes/macrophages, neutrophils, and T cells plays an important part in reshaping the tumor microenvironment and promotes metastasis at distant organs [20]. Taking advantage of the metastasis-orienting ability of specific types of cells, biomimetic strategies endow NDDSs with complicated biological functions and good biocompatibility, providing versatile options of constructing nanosystems eliciting antimetastasis effect. In the past decade, the field of treating metastatic breast cancer by bioinspired NDDSs has made a lot of progress, which will be illustrated in the following sections. In this chapter, three main approaches to developing bioinspired nanomedicine are introduced: coating nanoparticles with cell membranes, loading nanoparticles into living cells or fabricating nanoparticles using biomimetic materials.

\section{Cell membrane-coated NDDSs}

Abundant glycans, proteins and receptors on the cell membranes play an indispensable role in mediating biological functions. With well-developed cell membrane extraction technologies, the top-down membrane coating method has become the most intensively applied modality to prepare biomimetic NDDSs, which simplifies complex chemical modification [98]. Due to advantageous functions, numerous types of membranes have been exploited to fabricate biomimetic core-shell NDDSs for cancer therapy, including membranes of RBC (long circulation) [99], platelets (adhesion to damaged vessels) [100], bacteria (immunogenicity and hypoxia tropism) [101], cancer cells (homotypic binding and tumor-specific immune response stimulation) [102], and monocytes/macrophages (being recruited by tumors and metastases) [103], etc. 


\section{RBC membranes-coated NDDSs}

The RBC membrane presenting rich "self-markers" is widely used as a biomaterial for several properties that are favorable for drug delivery: (1) good biocompatibility, (2) elongated blood circulation, (3) enhanced tumor penetration, (4) easy availability in large-scale preparation $[99,104]$. However, tumor targeting and controlled drug release of RBC membrane-coated nanoparticles require combinational strategies.

Zhai et al. [74] used natural-source gelatin and RBC membranes to construct a cytotoxic $\mathrm{T}$ cell inspired nanovesicle (MPV) containing water-soluble drugs methylene blue and cisplatin for real-time tracking, light-controlled activation and cytoplasmic drug delivery. It was found that MPVs circulated in the blood for a long time and the photoacoustic properties of methylene blue in MPVs with a high concentration could be employed to realize realtime monitoring of drug accumulation in tumors. When MPVs highly accumulated in tumors, infrared laser irradiation was administrated to activate photodynamic/ photothermal effects to improve the penetration of MPVs in the tumor and facilitated the delivery of methylene blue and cisplatin to the cytoplasm of tumor cells. The process and extent of MPVs activation in tumors could be monitored in real time through photothermal imaging and fluorescence imaging. Under the synergy of photodynamic and chemotherapy, MPVs promoted tumor cell apoptosis, suppressed the primary TNBC tumor growth, and effectively inhibited the formation of lung metastatic nodules. This research constructed a visualizable real-time monitoring NDDS based on a cytotoxic T cell-mimicking strategy and provided a new idea for the effective treatment of metastatic TNBC [74].

Coating the drug-loaded micelles by RBC membranes can protect them from premature dissociation in blood. Heparan sulfate (HS), the substrate of heparinase (Hpa) can be an ideal candidate for synthesizing prodrugs to realized tumor cell-specific drug release, since Hpa is overexpressed in most advanced cancer cells $[105,106]$. In order to enhance the stability and increase the blood circulation time of micelles formed by HS-modified drugs, Lang et al. [107] used RBC membranes to wrap the HS-DTX conjugates micelles and obtained Hpa-sensitive biomimetic nanoparticles (rHS-DTX). The nanoparticle efficiently accumulated and specifically released DTX at the tumor sites with high expression of Hpa. rHS-DTX enhanced the accumulation of DTX in MCF-7 tumors and lung metastatic foci by 6.35 and 9.68 -folds respectively, achieving a striking tumor inhibition rate of $98.2 \%$ and pulmonary metastasis suppression rate of $99.6 \%$. rHS-DTX effectively improved the therapeutic effect of cytotoxic agents on breast cancer and reduced side effects. The potential value of Hpa-responsive NDDSs in the treatment of metastatic breast cancer was proved [107].

For overcoming the two main obstacles hampering sufficient drug accumulation in tumor cells, Su et al. [108] prepared the RBC membrane-coated PTX-loaded polycaprolactone (PCL) nanoparticles (RVPNs), which were administrated combining with a tumor penetration peptide, iRGD. The pharmacokinetic evaluation in rats presented that the half-life of RVPNs/iRGD in blood was $30.17 \mathrm{~h}$, which was significantly longer than that of the Taxol group $(1.93 \mathrm{~h})$. In the orthotopic 4T1 tumor mice model, the accumulation and penetration of RVPNs in primary tumors and metastatic sites were significantly increased. RVPNs/ iRGD significantly inhibited tumor growth by a ratio of $90 \%$ and inhibited about $95 \%$ of tumor lung metastasis [108]. Despite the potent anti-tumor and metastasis-inhibiting effect of RVPNs/iRGD, the drug release rate from RBC membrane was not sufficient for rapidly killing cancer cells. A light-sensitive nano-drug named PTX-PN@DiR-RV was constructed and expected to control the drug release making use of the cell membrane ablation effect of NIR laser. To achieve long circulation in the blood and metabolic distribution visualization, $\mathrm{Su}$ et al. [31] embedded the photosensitizer DiR into the RBC membrane, in which the PTX-loaded PCL nanoparticles were wrapped up. After accumulation in the tumor site, PTX-PN@DiR-RV could release PTX rapidly, which was triggered by NIR laserinduced photothermal effect. Under the synergistic effect of PTT and chemotherapy, PTX-PN@DiR-RV effectively killed cancer cells in the 4T1 orthotopic mammary tumor-bearing mice and inhibited the lung metastasis, serving as an in vivo tumor imaging probe simultaneously. PTX-PN@DiR-RV significantly increased the concentration of PTX in the primary tumors compared to Taxol, attributed to decreased blood clearance and enhanced tissue permeability resulted from laser-triggered hyperthermia. Taxol caused severe hepatotoxicity with structural and metabolic changes, while negligible abnormality of major organs was displayed in the PTX-PN@DiR-RV group due to the tumor-specific accumulation and permeation of PTX, suggesting the good biocompatibility of PTX-PN@DiR-RV. This research provided a promising strategy for the optimization of RBC-like NDDSs [31].

Although MSNs provide a flexible platform to deliver various therapeutic agents with high loading capacity, its aggregation in blood and limited circulation time restrict its application in fabricating NDDSs [109]. Su et al. [104] proposed a simple biomimetic strategy for MSN modification to elongate blood circulation time of MSN without 
diminishing its drug loading capacity, and employed laser irradiation to achieve tumor-specific drug release instead of ligand conjugation on surfaces. They constructed an MSN-supported RBC-camouflaged NDDS that was responsive to laser illumination. MSN loaded with $\mathrm{Ce} 6$ and DOX was encapsulated in RBC membranes to form RVscoated MSN-DOX/Ce6 (RMSN-DOX/Ce6). RBC membrane coating stabilized MSN in blood and prevented the encapsulated drugs from leakage. In virtue of the fluorescence of Ce6, RMSN-DOX/Ce6 acted as in vivo tumor imaging probe. Besides, when stimulated by an external laser, Ce6 generated ROS and destroyed the RBC membrane as well as the tumor cell membrane, leading to a rapid release and tumor-specific accumulation of DOX. Owing to the synergy of chemotherapy and PDT, RMSN-DOX/Ce6 efficiently inhibited the growth of primary breast cancer tumor and lung metastasis. In addition, since RMSN-DOX/Ce6 significantly increased the concentrations of DOX in tumors and decreased the distribution of DOX in hearts, no obvious signal of toxicity in mice was observed, offering a new direction of MSNs optimization for antimetastasis therapy [104].

\section{Cancer cell membrane-coated NDDSs}

The cancer cell membrane inherits the natural homotypic targeting ability of tumor cells, enlightening an intriguing approach to treating metastatic cancer [102]. Thus, cancer cells in primary tumors and metastatic foci could be targeted by cancer cell membrane-coated nanoparticles. The team designed several cancer cell membrane-derived biomimetic NDDSs that showed potential in suppressing both primary tumors and the metastatic lesions. CTCs construct the bridge between the primary tumor and metastatic tumors in distant organs [110]. In blood circulation, CTCs are prone to aggregation for prolonging the survival of metastatic cells and subsequently seed in the "soil" supporting the growth of metastatic foci [110]. Like other types of adhesion between living cells, homotypic aggregation of the CTCs is highly dependent on the multiple molecule-mediated cell surface interactions [102]. Sun et al. [19] anticipated that homotypic aggregation could be employed to target cancer cells, particularly those in dispersed metastatic nodules. Therefore, they constructed PTX-loaded PCL and pluronic F68 nanoparticles, which were coated with $4 \mathrm{~T} 1$ cell membrane (CPPNs) through the membrane fusion technology (Figure 5A). Membrane protein Thomsen-Friedenreich (TF) antigen, CD47 and E-cadherin which were crucial in homotypic aggregation were successfully transferred to the shells of the CPPNs. These shell-core structured cancer cell membrane-encapsulated nanoparticles with the mean diameter of $175.4 \pm 0.6 \mathrm{~nm}$ and slower blood clearance were enriched in primary tumors and metastatic sites of nude mice model bearing 4T1 cancer through homotypic targeting, thus increased the concentration of PTX in target sites (Figure 5B). As a result, CPPNs significantly inhibited tumor growth in situ and decreased the number of and lung metastatic nodules, revealing the value of biomimetic nanoparticles in anti-tumor metastasis (Figure 5C, D) [19].

A similar biomimetic strategy has been applied by the same group, in which gold nanocages (AuNs) were modified to prevent cargo leakage. The short circulation time and insufficient tumor targeting capacity limited the application of AuN as a high-efficient photothermal agent. Sun et al. [63] constructed light-sensitive core-shell biomimetic NDDSs with DOX-loaded AuNs as the inner cores and 4T1 cancer cell membranes as the outer shells (CDAuNs). Owing to the natural properties of cancer cell membranes, CDAuNs achieved long circulation in the blood and were enriched in all the colonization of cancer cells via homotypic targeting-mechanism. Under infrared light irradiation of $808 \mathrm{~nm}$, CDAuNs increased the temperature in the tumor site and promoted the burst release of DOX. CDAuNs combined PTT and chemotherapy to effectively inhibit tumor growth and metastasis. Compared with free DOX, CDAuNs with or without NIR laser irradiation lowered the DOX concentrations in heart tissues by more than three times, resulting significant relief of cardiotoxicity. This personalized cancer therapy strategy with good biocompatibility in vivo showed potential in clinical applications [63].

\section{Macrophage membrane-coated NDDSs}

Macrophages play important roles in tumor progression and metastasis. For instance, macrophages can bind metastatic cancer cells, supporting the survival of metastatic cancer cells and growth of metastatic lesions. Therefore, macrophage membrane coating has potential in targeting metastatic cancer cells [111]. Cao et al. [75] used acid-sensitive phospholipids 1,2-dioleoyl-sn-glycero3-phosphoethanolamine (DOPE) and DSPE-PEG2000 to prepare acid-sensitive liposomes encapsulating the chemotherapeutic drug maytansine derivative DM1 (EL). Then, core-shell biomimetic nanoparticles (MEL) were constructed by coating EL with the mouse monocyte/ macrophage membrane via the extrusion method. MEL presented $\alpha 4$ integrins of the macrophage and VCAM-1 which could significantly increase the uptake into breast 

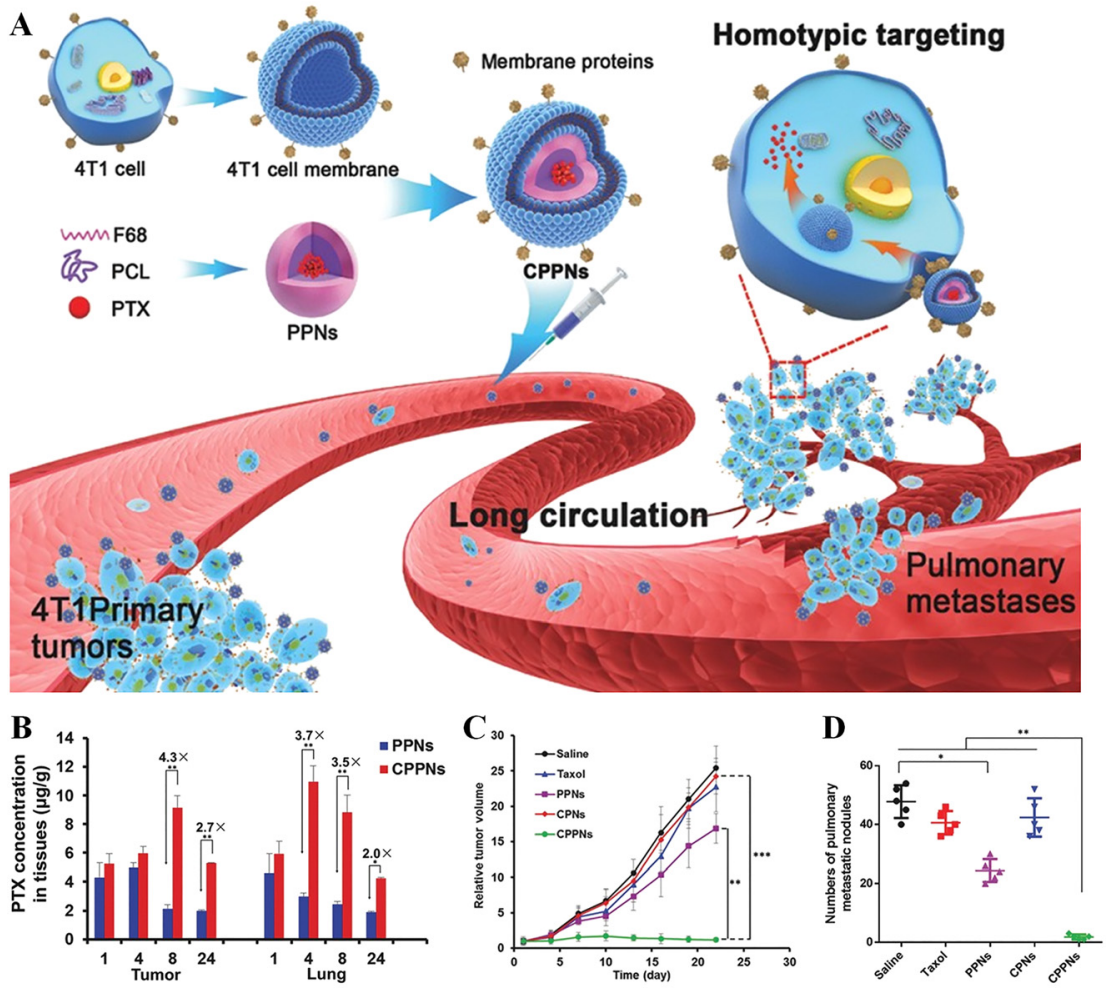

Figure 5: Cancer cell membrane-coated biomimetic nanoparticles for targeted therapy of homotypic tumors. (A) Schematic illustration of the preparation and mechanisms of the cancer cell membrane-coated nanoparticles for treatment of metastatic cancer. (B) Quantitative analysis of the PTX distribution in tumors and lungs of delivered with PPNs or CPPNs. (C) Tumor growth curves of each group. (D) Quantitative analysis of pulmonary metastatic nodules of each group. Data are given as the mean \pm SD $(n=5)$. Statistical significance: ${ }^{*} p<0.05,{ }^{* *} p<0.005$, and ${ }^{\star \star \star} p<0.0005$. CPN: Cancer-cell-biomimetic vesicles coated polymeric nanoparticles; CPPN: Cancer-cell-membrane coated paclitaxel-loaded polymeric nanoparticles; PCL: poly(caprolactone); PPN: Paclitaxel-loaded polymeric nanoparticles; PTX: paclitaxel; SD: standard deviation. Reproduced with permission [19]. Copyright 2016, Wiley-VCH.

cancer cells. In breast cancer mice model with lung metastasis, spectrum/CT and immunofluorescence imaging confirmed that MEL efficiently targeted and inhibited lung metastasis. This proof-of-concept study confirmed the efficacy of macrophage-biomimetic strategy for specific targeting to lung metastasis via a well-established procedure [75].

\section{Cell-delivered NDDSs}

Although the cell membrane-coating strategy has been studied intensively, the preparation procedure is laborious with inevitable loss of biological functions. Therefore, loading nanoparticles in living cells can be a rational approach. The capacity of capturing a large variety of exogenous substances makes monocytes/macrophages potential carriers of NDDSs [112]. Monocytes can be recruited to the tumor sites and differentiated into macrophage, affecting the formation of metastatic foci [103, 113]. Making use of these unique abilities of monocytes/macrophages, several monocytes-based drug delivery vehicles to target primary tumors and metastases have been constructed. In order to ensure the vitality of living cells during drug delivery, preventing unmatured drug release and controlling the activation of intracellular cargos precisely are of great importance.

Monocytes with an upregulated Ly6C expression are increased and recruited to the tumor tissue during cancer progression [113]. Lang et al. [64] co-cultured monocytes isolated from peripheral blood with acid-sensitive BD-PEG micelles loaded with the chemotherapy drug PTX to obtain a monocyte-micelle system (PM@MC). Before PM@MC reaching tumor cells, PM packed with PTX stayed quiescent to avoid poisoning monocytes. After exposed to the acid environment inside endosomes/lysosomes in tumor cells, the dissociation of PM would occur. Due to the recruitment of monocytes by tumors, the monocyte-micelle 
system could actively migrate to the tumor site, following the signal from inflammatory factors. PM@MC enhanced the penetration and accumulation of PTX in the primary tumor and pulmonary metastatic nodules, which effectively inhibited the growth of cancer cells. The PTX amount in tumors and lungs were increased by 15.1-fold and 11.6-fold respectively, with $99.2 \%$ of lung metastatic nodules inhibited. Taxol caused modest hepatic injury and bone marrow suppression, while PM and PM@MC had good biocompatibility [64]. Based on the tumor-targeting capacity of Ly6C ${ }^{\text {hi }}$ monocytes, Lang et al. [114] recently designed another bioinspired drug delivery system to achieve the synergy of chemotherapy and immunotherapy. HY19991 (HY) was encapsulated into micelles formed by two Hpa-sensitive amphiphilic prodrugs, HS-DTX and HS-NLG919 conjugates (HS-NLG) (HDNH, Figure 6A). Then, HDNH was loaded into the Ly6 $\mathrm{C}^{\text {hi }}$ monocyte isolated from the peripheral blood through phagocytosis. HDNH@MC exhibited selective cytotoxicity for breast cancer cells but not normal breast epithelial cells. NLG919 could suppress IDO signal pathways and decrease the Tregs number, increasing the proportion of $\mathrm{CD}^{+}{ }^{+} \mathrm{T}$ cells. HY19991 inhibited the PD-1/PD-L1 interaction and relieved the constraint on $\mathrm{CD}^{+} \mathrm{T}$ cells (Figure 6B-D). As a result, HDNH@MC
A

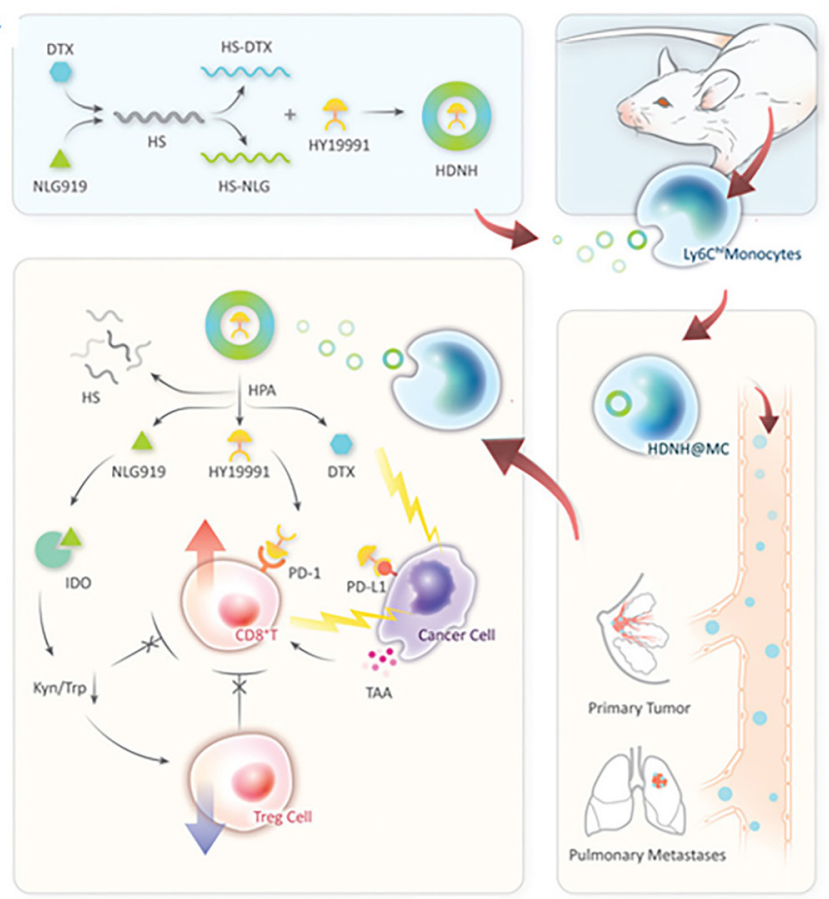

E

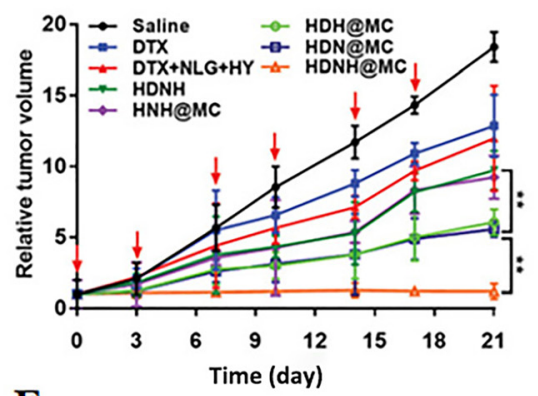

F

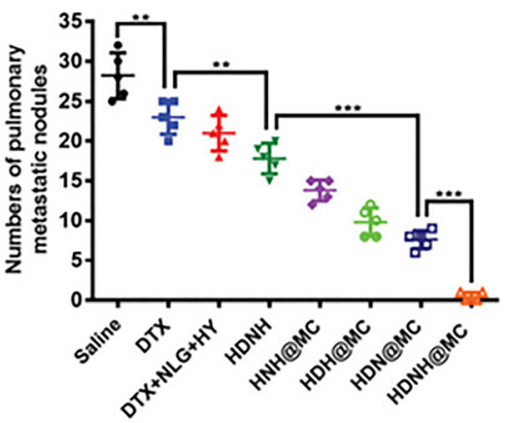

D

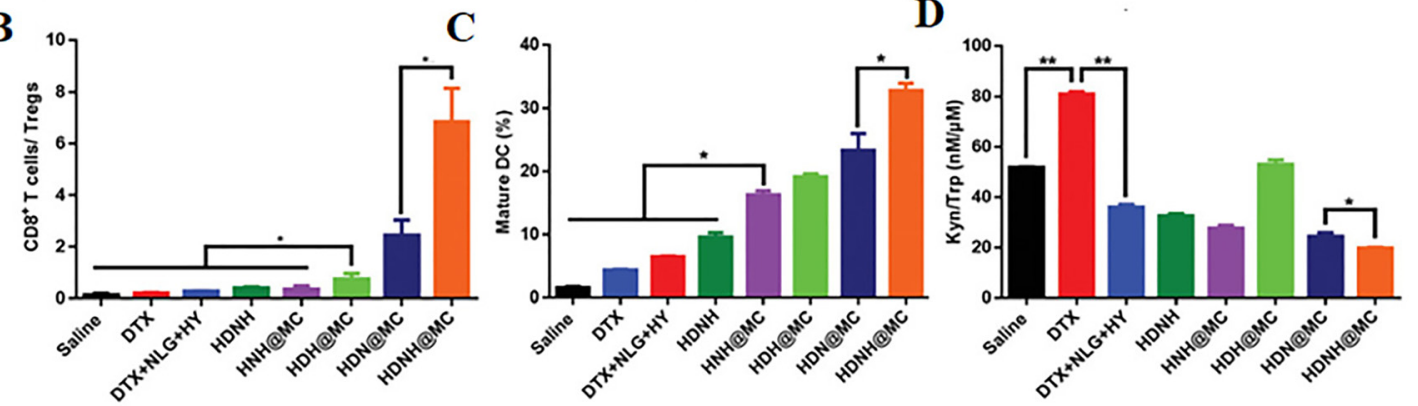

Figure 6: Ternary regulation of tumor microenvironment by Hpa-sensitive micelles-loaded monocytes improves chemo-immunotherapy of metastatic breast cancer. (A) Chemo-immunotherapy of metastatic breast cancer by the Hpa-sensitive micelles@monocytes system. (B) The ratio of $\mathrm{CD}^{+} \mathrm{T}$ cells to Tregs in tumors measured by flow cytometry. (C) The proportion of mature dendritic cells in tumors examined by flow cytometry. (D) The ratio of Kyn to Trp in tumors examined by HPLC-MS. (E) The variation curves of tumor volumes during the therapy period. (F) Numbers of the lung metastatic nodules. Data are shown as mean \pm SD $(n=5)$. ${ }^{*} p<0.05$ and ${ }^{\star *} p<0.01$. DTX: Docetaxel; HS: heparan sulfate; HY: HY19991; NLG: NLG919; HDH: HY-loaded HS-DTX conjugate micelle; HDN: HS-DTX/HS-NLG conjugate hybrid micelle; HDNH: HY-loaded HS-DTX/ HS-NLG conjugate hybrid micelle; HPA: Heparanase; HPLC-MS: high performance liquid chromatography-mass spectrometry; IDO: Indoleamine 2,3-dioxygenase; MC: monocytes; PD-1: Programmed cell death-1/; PD-L1: Programmed cell death-ligand 1; SD: standard deviation; TAA: Tumor-associated antigen; Treg: Regulatory T cells. Reproduced with permission [114]. Copyright 2020, Wiley-VCH. 
elicited improved tumor-inhibition effects compared with free drugs on $4 \mathrm{~T} 1$ breast carcinomas mice model, with less lung metastasis and prolonged survival of mice (Figure 6E, F) [114].

The differentiation of monocytes to macrophages at tumor sites can be exploited as a drug release stimulus [115]. For instance, legumain is abnormally overexpressed in the differentiated macrophages $[103,115]$. Based on this mechanism, He et al. [29] designed legumain-motivated nanoparticles loaded in inflammatory monocytes to actively target lung metastasis. They synthesized a legumainsensitive polystyrene maleic anhydride-alanine-alanineasparagine-maytansine conjugate (SMA), which could self-assemble in water to form nanoparticles (SMNs). The SMNs were incubated with and loaded into monocytes, forming an active targeting enzyme-sensitive intelligent delivery system (M-SMNs). Owing to the sensitivity to inflammatory factors in the primary tumors and metastatic lesions, M-SMNs were effectively distributed to lungs and further migrated to the lung metastatic foci. After monocytes differentiated into macrophages, the anticancer drugs were released in the state of free drug molecules along with nanometer-sized vesicles. M-SMNs efficiently inhibited the proliferation, migration, and invasion of metastatic 4T1 cancer cells. In lung metastatic breast cancer model, M-SMNs significantly improved the targeting delivery to lung metastasis and the tumor penetration of cytotoxic agents, with a lung metastasis inhibition ratio of $77.8 \%$ [29].

Cao et al. [30] have constructed a bioengineered macrophage-based drug delivery system (LD-MDS), which could effectively target tumor metastases and then intelligently transformed into nanovesicles and secondary nanovesicles for anti-metastatic therapy (Figure 7A). This system anchored legM and redox-sensitive prodrug of cytotoxic soravtansine (DM4) on the surface of living macrophages to form LD-MDS. At the site of metastasis, LD-MDS was specially activated by legumain, thus increased the penetration of LD-MDS into metastatic lesions (Figure 7B). LegM transformed to melittin which facilitated the transformation of LD-MDS into DM4-loaded exosome-like nanovesicles (DENs), promoting cell death in metastases. Subsequently, damaged tumor cells released secondary nanovesicles and free drugs to destroy neighboring tumor cells. In vivo studies showed that this system significantly inhibited lung metastasis (Figure 7C, D). This study provided a new idea for exploring endogenous macrophages as live cell drug delivery vehicles targeting tumor lung metastasis [30].

\section{Endogenous nanostructure-inspired NDDSs}

The dormant stage before outgrowth of cancer cells is a promising anti-metastatic therapeutic entry point $[6,35]$. At this stage, CSCs are responsible for the initiation of metastasis in distal organs, indicating the importance of eradicating those special cancer cells in dormant tumors as a strategy to prevent metastasis [110]. However, CSCs usually live in tumor internal zones away from tumor vasculature, and efficient drug delivery to CSCs requires the deep penetration across physical barriers of solid tumors [116]. A cage-like nanostructure which has potential in drug delivery is formed by ferritin, a ubiquitous endogenous iron storage protein. Ferritin has an outer diameter of $12 \mathrm{~nm}$ and a cavity with the inner diameter of $8 \mathrm{~nm}$, and can be developed to NDDSs for tumor imaging and therapy. Since ferritin is able to bind overexpressed transferritin receptor 1 (TfR1) and scavenger receptor class A membrane 5 (Scara5) on cancer cells, it can be employed to achieve tumor-targeting and deep penetration in tumor tissues. Tan et al. [76] designed and established a ferritin biomimetic nanocage system containing the photothermal therapeutic agent DiR and chemotherapeutic epirubicin to achieve efficient drug delivery to CSCs (Figure 8A). The DiR-loaded biomimetic nanocages (DBN) could be largely internalized by cancer cells and preferentially localized ALDH ${ }^{\text {high }}$ CSCs cohorts (Figure 8B). The precise PTT effects induced by DBN overcame the nanoparticle-trapping effect caused by tumor-related macrophages and fibroblasts. Epirubicinloaded ferritin nanocages (EBN) could penetrate into deep tumor tissues to exert sufficient therapeutic effects (Figure 8C). Combining PTT and chemotherapy, the ferritin-based biomimetic nanocage system significantly inhibited lung metastasis of breast cancer (Figure 8D-F). This work proposed a new strategy for anti-tumor metastasis by targeting CSCs in tumor tissues to inhibit breast cancer metastasis [76].

In blood circulation, nanoparticles can adsorb various plasma proteins to form a protein corona, and human serum albumin (HSA) is one of the mostly detected proteins in the naturally-formed corona around nanoparticles [117]. Besides a long half-life of more than ten days in blood, HSA also preferentially accumulates in tumors due to its role as an amino acid provider. Accordingly, Cao et al. [77] anticipated that nanosystems masked by the corona formed by HSA would prolong blood circulation time and enhance the capacity of tumor targeting and perfusion, which were in favor of the drug delivery against metastatic breast 
A

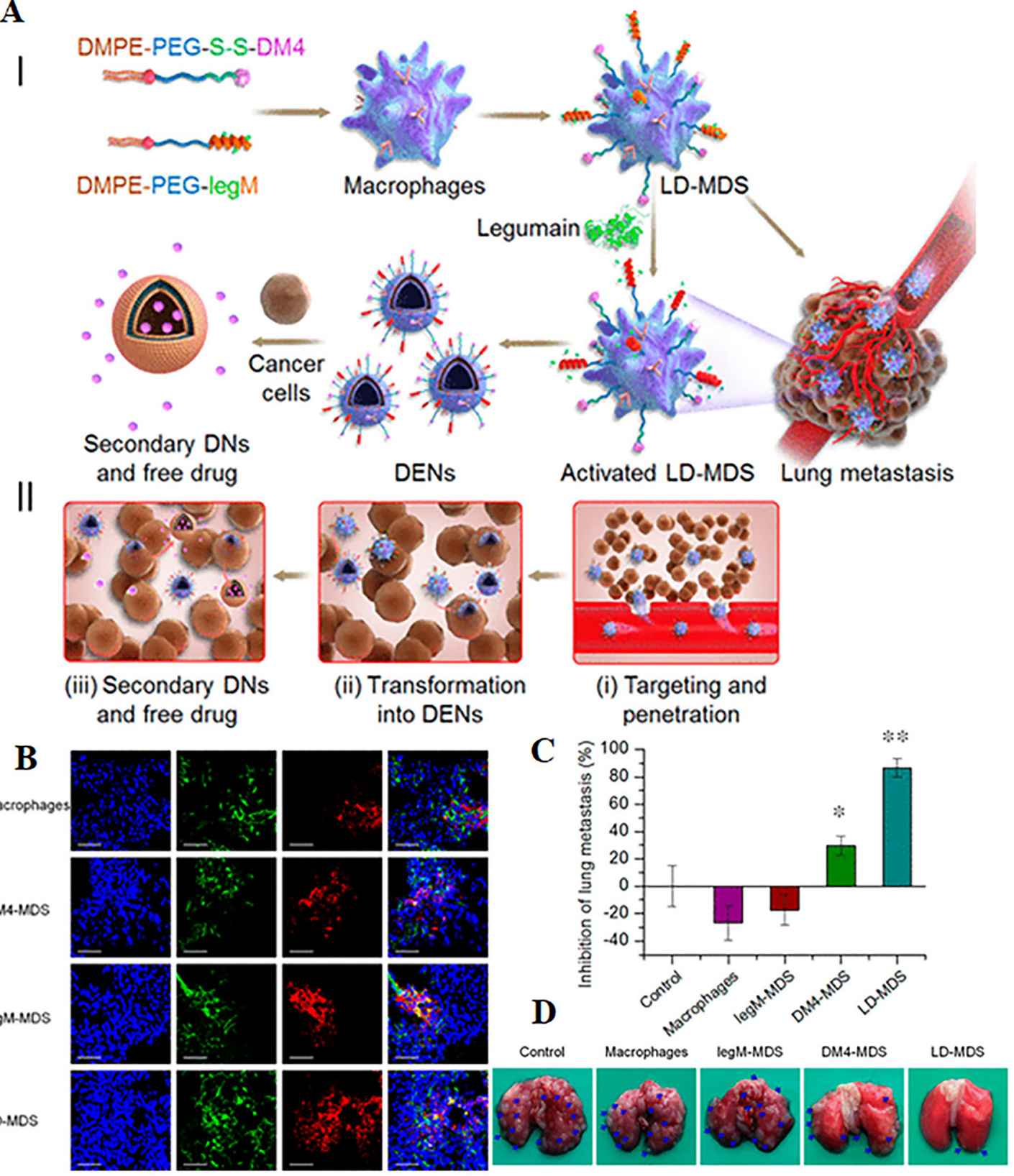

Figure 7: Bioengineered macrophages targeting lung metastasis. (A) The LD-MDS is preferentially recruited to lung metastases and responsively transformed into nanovesicles and secondary nanovesicles for an effective anti-metastasis therapy. (I) Fabrication of the LD-MDS and its transformation into nanovesicles in response to legumain protease; (II) the specific targeting of LD-MDS for lung metastases and drug delivery to cancer cells. (B) The deep penetration of the LD-MDS in metastatic lesions in lungs, scale bar $=50 \mu \mathrm{m}$. (C) The relative inhibition rate of various MDSs compared to that of the saline group, ${ }^{*} p<0.05,{ }^{\star *} p<0.01$; (D) typical lung tissue images with visualized metastatic nodules (blue arrows). DEN: Soravtansine-loaded exosome-like nanovesicles; DM4: Soravtansine; DMPE: 1,2-Dimyristoyl-sn-glycero-3-phosphoethanolamine; LD-MDS: Melittin and soravtansine-loaded macrophage-based delivery system; MDS: Macrophage-based delivery system; PEG: poly(ethylene glycol). Reproduced with permission [30]. Copyright 2018, American Chemical Society.

cancer. They firstly conjugated R9 with DOPE via disulfide bonds to form an amphiphilic/reduction-sensitive peptide derivative (R9-S-S-DOPE). Then, DOPE, R9-S-S-DOPE, IR-780 and Twist siRNA were combined to form a cellpenetrating dual prodrug delivery system (DRI-S). DRI-S was modified with HSA (DIR-S@HSA) subsequently. Due to the protection and tumor-targeting effect of albumin, DRI-S@HSA showed extended blood circulation time, and effectively accumulated at the tumor metastasis site. DRI-S@HSA also achieved stronger tumor penetration and 
A

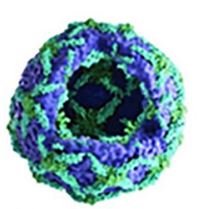

Nanocages

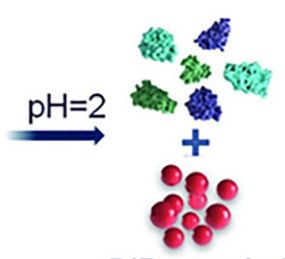

DiR or epirubicin

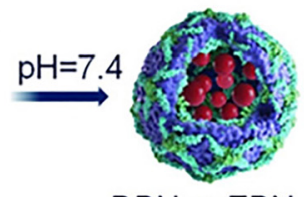

$D B N$ or $E B N$

NIR
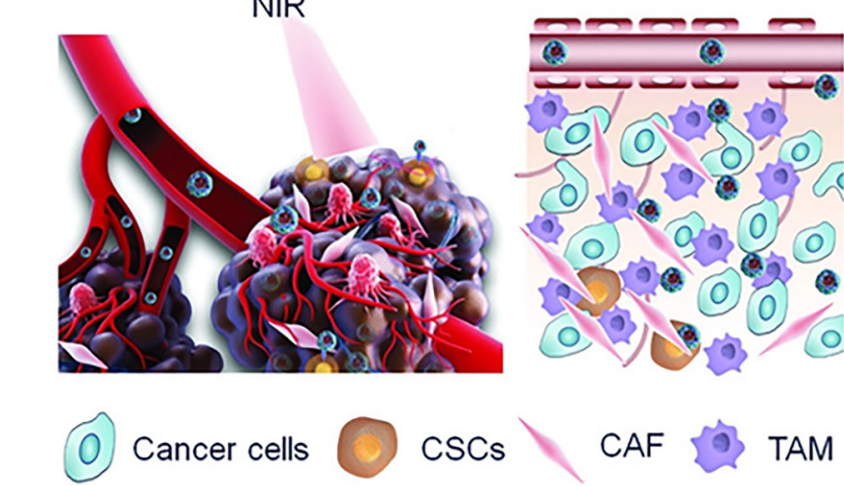

D

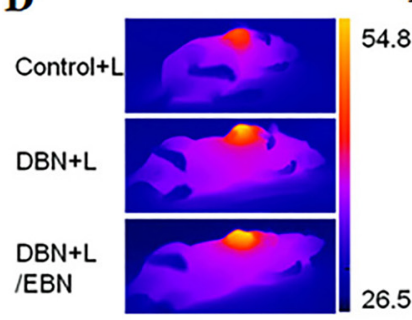

B

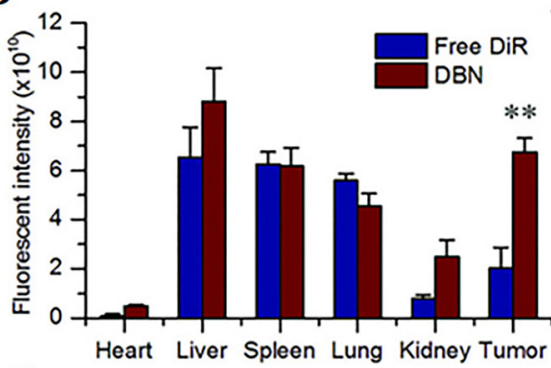

C DAPI
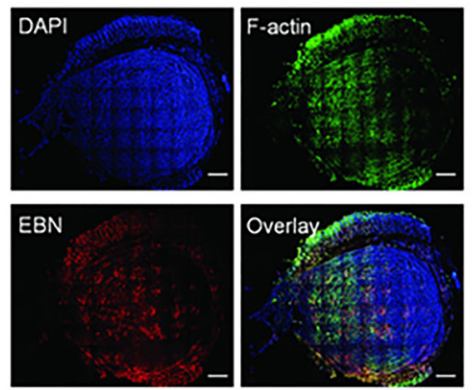

TAM

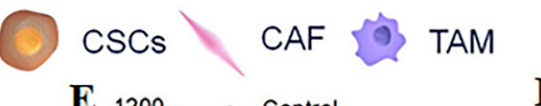

F

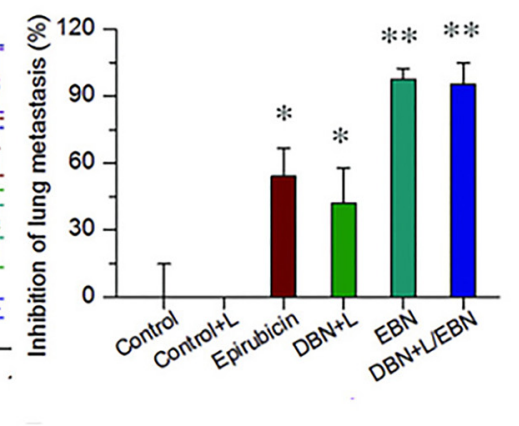

Figure 8: Deep tumor-penetrating nanocages improve accessibility to cancer stem cells for photothermal-chemotherapy of breast cancer metastasis. (A) Schematic illustration of deep tumor-penetrating biomimetic nanocages with preferential CSCs-accessibility for effective antimetastasis therapy. The biomimetic nanocages can effectively deliver the photothermal agent DiR and cytotoxic epirubicin to CSCs in tumor mass for photothermal-chemotherapy of breast cancer metastasis. (B) The quantified distribution of DBN and free DiR in major organs at $4 \mathrm{~h}$ post injection, ${ }^{* *} \mathrm{p}<0.01$. (C) The permeation of EBN in the whole tumor mas under laser confocal scanning microscopy (LCSM), scale bar=1.0 mm. (D) The typical thermal images of tumor-bearing mice. (E) The tumor growth profiles of each group. (F) The inhibition rate of lung metastasis of each group, ${ }^{\star}<<0.05$, ${ }^{* *}$ p<0.01. CAF: Cancer-associated fibroblasts; CSC: Cancer stem cells; DAPI: 2-(4-amidinophenyl)-6-indolecarbamidine dihydrochloride; DBN: 1,1-Dioctadecyl-3,3,3,3-tetramethylindotricarbocyanine iodide; DiR: 1,1-Dioctadecyl-3,3,3,3-tetramethylindotricarbocyanine iodide; EBN: Epirubicin; TAM: Tumor-associated macrophages. Reproduced with permission [76]. Copyright 2018, Wiley-VCH.

effectively inhibited the growth of tumor and the formation of lung metastasis, which proved the potential of this biomimetic tumor-targeting drug delivery strategy in antimetastatic treatment [77].

High-density lipoproteins (HDLs) are endogenous nanostructured particles composed of diverse proteins and lipids, playing an important role in transporting signaling lipids, proteins, and endogenous microRNAs to recipient cells [118]. HDLs have specific properties which make them as ideal nanoplatforms to deliver various therapeutic agents to tumors: (1) extended period of blood circulation time, (2) ultra-small sizes (10-20 nm) ensuring efficient accumulation in tumor sites and penetration throughout tumor regions, (3) recognition of the cholesterol transporter scavenger receptor class B type I (SR-BI) overexpressed on tumor cells [119]. CAFs and TAMs, as pivotal constructors of tumor stroma, seriously hinder the tumor penetration of nanomedicine, indicating the importance of remodeling the tumor stromal microenvironments (TSM) to promote accessibility of nanoparticles to cancer cells [6]. Tan et al. [78] prepared a bioinspired lipoprotein (bLP) nanosystem that was loaded with either a photothermal agent DiR (D-bLP) or the anticancer drug mertansine (M-bLP). The first wave of D-bLP was aiming at 


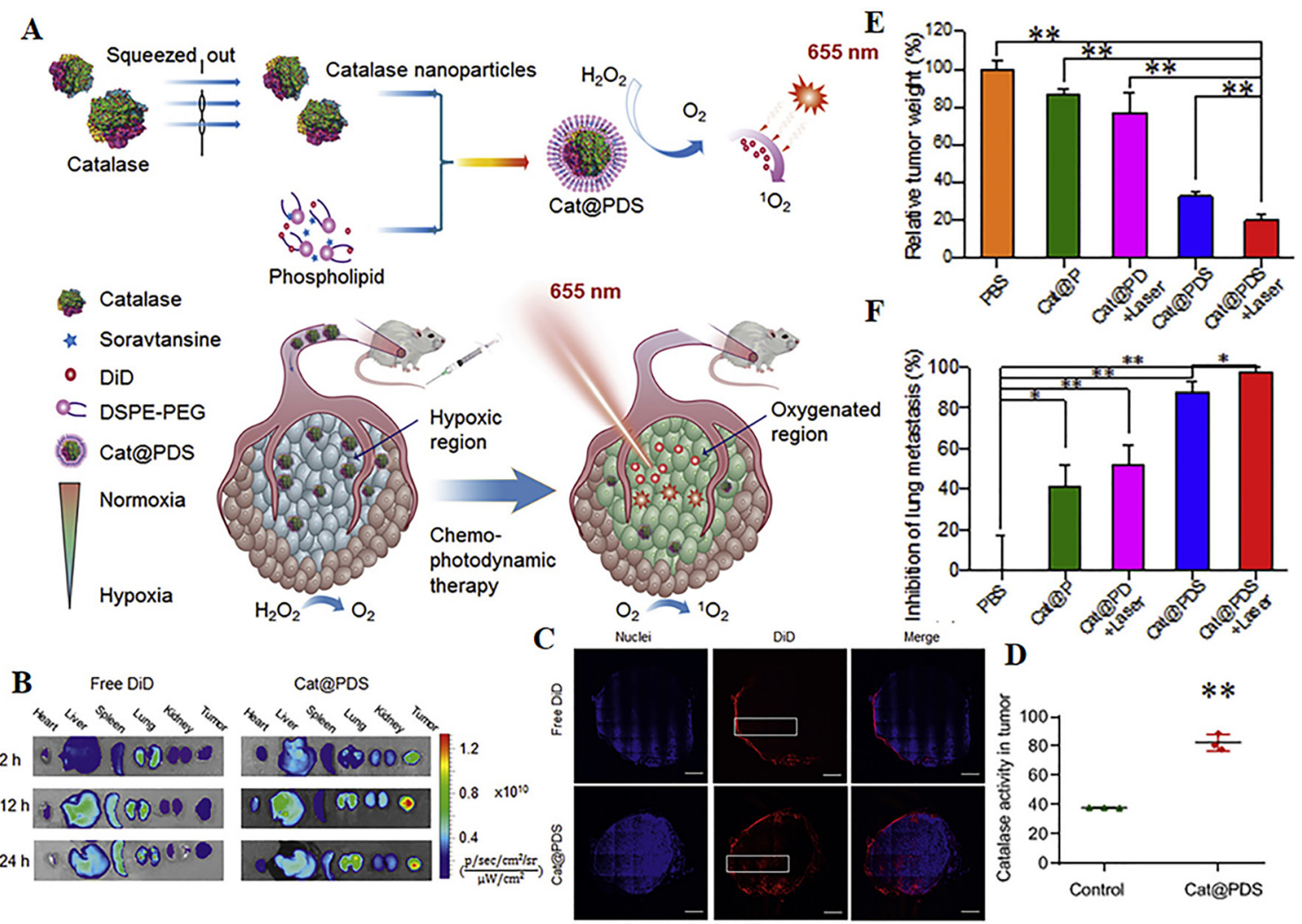

Figure 9: The PEGylated phospholipids membrane-coated nanocatalase system for chemo-photodynamic therapy. (A) Schematic illustration of phospholipid membrane-coated nanocatalase system (Cat@PDS) to relieve tumor hypoxia for enhanced chemo-photodynamic therapy. (B) The ex vivo imaging of Cat@PDS in major organs at different time points after injection. (C) The intra-tumoral permeation of Cat@PDS (red) under LCSM, scale bar=1 mm. (D) The catalase activity in tumors of the Cat@PDS group. Data are mean \pm SD $(n=3)$, ${ }^{* *} p<0.01$. (E) The relative tumor weights. Data are mean $\pm S D(n=5),{ }^{* *} p<0.01$. (F) The inhibitory rate of lung metastasis. Data are mean $\pm S D(n=5),{ }^{*} p<0.05,{ }^{* *} p<0.01$. Cat: Catalase; DSPE: 1,2-Distearoyl-sn-glycero-3-phosphoethanolamine; LCSM: Laser confocal scanning microscope; PDS: DiD and soravtansine embedded in the phospholipid membrane of 1,2-distearoyl-sn-glycero-3-phosphoethanolamine- $N$-[methoxy(polyethylene glycol)-2000]; PEG: poly(ethylene glycol); SD: standard deviation. Reproduced with permission [79]. Copyright 2020, Elsevier.

remodeling TSM barriers and clearing up the intratumoral delivery obstacles via the photothermia effect to facilitate the tumor penetrating of the following administrated M-bLP. They proved that D-bLP administration with laser irradiation remarkably reduced CAFs and TAMs proportions and down-regulated collagen I and fibronectin concentrations in tumor stroma. Thus, the bLPbased TSM remodeling strategy significantly enhanced the accessibility towards cancer cells, resulting in $95.9 \%$ inhibition of tumor growth and $97.4 \%$ suppression of lung metastasis of 4T1 tumor [78].

Hypoxia status in tumors contributes to the metastasis and drug resistance of cancer cells, as well as diminishes the efficacy of PDT. Decomposing high concentration of $\mathrm{H}_{2} \mathrm{O}_{2}$ in the tumor microenvironment into oxygen can be a potential strategy to increase the intra-tumoral oxygen levels. Catalase (CAT) from mammalian erythrocytes has high enzyme activity of converting $\mathrm{H}_{2} \mathrm{O}_{2}$ into water and oxygen, and can be modified onto nanoparticles [120].
Therefore, reserving the biological activity of CAT nanoparticles and enhancing their permeation into deep hypoxic regions are of great importance for effective tumor oxygenation. Inspired by the structure of erythrocytes, the natural carrier of CAT, Yin et al. [79] prepared a PEGylated phospholipids membrane-coated nanocatalase system with long-circulation and deep penetration into tumors (Figure 9A). The photodynamic agent DiD and a cytotoxic agent soravtansine were embedded in the DSPE-PEG phospholipid membrane to prepare drug-loaded coating for the encapsulation of CAT nanoparticles. With enhanced permeation and accumulation at tumor sites, the nanocatalase system relieved hypoxia status and improved the therapeutic efficacy of PDT under laser irradiation of $655 \mathrm{~nm}$ (Figure 9B-D). As a result, the tumor oxygenationaided chemo-photodynamic therapy induced significant inhibition of 4T1 tumor growth and striking $97.2 \%$ suppression of lung metastasis in mice (Figure 9E, F) [79]. 


\section{Large-scale production of biomimetic NDDSs}

Despite of substantial success in laboratory studies during last decades, several problems are required to be resolved for further clinical translation of biomimetic nanomedicines. Considering those outer materials of biomimetic NDDSs originate from the animals themselves, they usually possess good biocompatibility and low immunogenicity. Nevertheless, it must be noticed that some cellular components or secretions might be toxic or influence the intercellular communications among tumor cells, stromal cells and immune cells [121]. Moreover, rigorous protocols and safeguards should be obeyed during the manufacture process to avoid biohazard [122]. Current preparation methods of biomimetic nanomedicines are complicated with lower productivity and higher costs than the synthetic nanocomposites. In contrast to synthetic nanomaterials, autologous cell components are rare and precious, and the quality of these materials should not be compromised during the scale-up production. Exploring preparation methods suitable for industrial manufacture will largely promote the clinical translation of biomimetic nanomedicines. For instance, the physical extrusion method is the mostly used method to prepare cell-membrane-coated NDDSs, although inefficient for scale-up production [123]. Recently, microfluidic electroporation and lyophilization have been used to construct the cell membrane-coated nanoparticle structures, achieving higher throughput and preserving the membrane functions well during production processes [124]. Advances in nanotechnology and following the guidance of good manufacturing practice (GMP) will push biomimetic nanotechnology closer to clinical applications.

\section{Other materials}

\section{Hydrogel}

Surgical resection of solid tumors usually leaves residual microtumors which probably causes lethal tumor recurrence and metastasis [125]. Though in situ administration of tumor vaccine have induced encouraging clinical response, the inefficiency to co-deliver multiple antigen peptides and adjuvants and the immunosuppressive tumor microenvironment remained to be overcome. Wang et al. [22] isolated single tumor cells from transplanted tumors of tumor-bearing mice and loaded the inactivated tumor cells with the immunomodulatory molecule JQ1 (a BRD4 inhibitor) and the photothermal molecule ICG. These drug-carrying tumor cells along with tumor-penetrable peptides were incorporated into temperature-sensitive hydrogels, thereby a personalized tumor vaccine (PVAX) was prepared. PVAX was proved to realize highly efficient photothermal conversion, produce photothermal effects, and stimulate the release of JQ1 and tumor-associated antigens (TAA). The released TAA could promote the maturation of dendritic cells and the infiltration of $\mathrm{CD} 8^{+} \mathrm{T}$ cells in tumor tissues, while JQ1 significantly down-regulated the expression of PD-L1 in tumor tissues, blocking the PD-1/PD-L1 immune checkpoint pathway and enhancing the anti-tumor immune response effect of PVAX. At the same time, PVAX also activated tumor-specific immune memory effects and inhibited the recurrence and metastasis of breast cancer after surgery. This tumor vaccine provided new ideas for personalized immunotherapy after tumor surgery [22].

\section{Inorganic nanoparticles}

Inorganic nanomaterials can be exploited to produce NDDSs due to the distinct physicochemical properties and the expandable production scale [126]. Gold nanoparticles are known as bio-inert and non-toxic nanocarriers with high photothermal conversion efficiency. Wang et al. [80] constructed DNA-entangled gold nanorods loaded with DOX (GNR@DOX) for the treatment of metastatic breast cancer.GNR@DOX could exert efficient photothermal ablation (PTA) of tumors and promote the therapeutic effect of chemotherapy under NIR irradiation. In mice bearing 4T1 metastatic breast tumors, GNR@DOX promoted tumor cell apoptosis and shrank the blood vessels at the tumor sites, inhibiting the growth of primary tumors and suppressing lung metastasis [80].

Biocompatible MSNs possess various advantages in line with the requirements of drug delivery, including ordered porous structures, well-controlled particle and pore sizes, high drug-loading capacity, large surface area and variety of surface modification [127]. These properties make MSNs suitable for the delivery of iron oxide nanoparticles, which are good candidates as contrast agents for T1-weighted magnetic resonance imaging (T1-MRI). Wu et al. [81] constructed a pH-responsive theranostic nanosystem by incorporating cytotoxic DOX into ironcontaining MSNs via metal-ligand coordination bonding. The DOX bound to the iron through chelation was quickly released under acidic conditions. As drug left the mesopore channels gradually, iron paramagnetic centers were exposed synchronously with T1-MRI of the nanomedicine, achieving the real time monitoring of the release of anticancer drugs. After intravenous administration, the 
nanosystem could be enriched at the tumor site and released DOX in the acidic tumor environment and inside tumor cells. Meanwhile, it enhanced anti-tumor efficacy and inhibited the lung metastasis through downregulating metastasis-related proteins [e.g., urokinase plasminogen activator (uPA), MMP2 and cyclooxygenase-2 (COX-2)] and reversed MDR via suppressing the drug efflux pump P-glycoprotein [81].

In the further cooperated research, mesoporous carbon nanoparticles (MCNs) with well-defined morphology, high hydrophilicity and dispersity are fabricated for cancer therapy. Chen et al. [128] constructed organic/inorganic hybrid MSNs to deliver silibinin. This nano-drug could significantly inhibit the migration and invasion of metastatic breast cancer cells MDA-MB-231. Later, Chen et al. [129] constructed hollow-structured mesoporous silica nanoparticles (HMCNs) with a red blood cell-like shape, which could be used for the efficient delivery of DOX. The oxidization with concentrated $\mathrm{HNO}_{3}$ and $\mathrm{H}_{2} \mathrm{SO}_{4}$ endow $\mathrm{HMCNs}$ with high hydrophilicity. The red blood cells-simulating shape facilitated the transportation of NPs in blood vessels compared with the spherical counterparts. After entering tumor cells, DOX was released in acid lysosomes to inhibit growth, migration and invasion of tumor cells [129].

Although having advantages such as high adsorption capacity, good biocompatibility, easy access to chemical modification and physical attachment, etc., the problem of aggregation limits the application of nanodiamonds. Xiao et al. [130]. constructed a water-soluble and longcirculating nanodiamond-mediated drug delivery system, in which the nanodiamond was loaded with DOX via physical adsorption and coated by DSPE-PEG to increase the dispersibility. This system had good compatibility and delivered DOX into tumor cell nucleus after intravenous administration. It effectively accumulated in the lung tumor metastasis, thereby inhibiting the lung metastasis of the $4 \mathrm{~T} 1$ breast cancer [130].

\section{Liposomes}

Liposomal formulations are highly potential for the delivery of chemotherapeutic drugs due to high loading capacity, uncomplicated preparation procedure, and low systemic toxicity. Feng et al. [23] synthesized a kind of clinically translatable phospholipid-mimic OXA prodrug (Oxalipid), which could self-assemble to form a liposomal nanostructure, with the drug loading capacity as high as $27 \%$ and the average particle size of $81.8 \pm 2.1 \mathrm{~nm}$. Compared with free OXA, OXA prodrug liposomes showed longer blood circulation time, increased tumor accumulation, and significantly inhibited the progression of metastatic triplenegative breast cancer. The research results demonstrated the advantages of phospholipid-mimic prodrugs in the treatment of metastatic triple-negative breast cancer [23].

\section{Conclusion and perspectives}

Metastatic breast cancer has become the first killer of women worldwide. Unfortunately, current therapeutic modalities show only moderate enhancement of patient survival. In the past two decades, NDDSs developed rapidly as potential platforms for anti-metastasis therapy. However, clinical transformation of NDDSs has encountered with a series of challenges including limited penetration in primary or distant tumors, insufficient efficacy due to the heterogeneity biology of tumors and the difficulty of production in large scale. Considering these key problems, our team focus on enhancing tumor accumulation and penetration of NDDSs to improve the outcome of metastatic breast cancer treatment. To achieve this goal, three strategies have been employed to construct antimetastasis NDDSs: (1) Optimizing physicochemical properties, (2) Remodeling tumor microenvironment, (3) Taking advantage of endogenous nanostructures and cells with intrinsic tumor-targeting character. The team exploited the drug delivery potential of various materials including polymeric micelles and nanoparticles, liposomes, inorganic nanoparticles, endogenous nanostructures and living cells. To realize the tumor site-specific and programmed drug release, multiple stimuli-responsive moieties have been ingeniously incorporated into one drug delivery system. For instance, the sensitivity to endogenous stimuli (acidity, redox, enzymes etc.) efficiently increases the concentrations of active drugs in primary and metastatic tumors, thus eradicates cancer cell colonization and reverses multi-drug resistance. Responsiveness to exogenous stimuli like light and heat ensures the enhanced permeability of solid tumor tissues for NDDSs and precise control of drug release under the guide of tumor imaging, indirectly inhibiting the occurrence of metastasis through smashing primary tumors. Moreover, a series of biomimetic NDDSs which can be classified into tumor-orienting cells (monocytes, macrophages, tumor cells)-inspired drug delivery systems and endogenous nanostructures (lipoproteins, ferritins, nanocatalase)-based nanocarriers have been reported. Biomimetic NDDSs provided highly bio-compatible platforms for personal treatment of metastatic cancer.

Although functional NDDSs have achieved unprecedented development, there are still lots of problems to resolve. First, comprehensive safety of cancer treatment 
based on NDDSs should be evaluated cautiously. The introduction of exogenous materials may cause harmful immunological response and long-time toxicity. Nowadays, artificial intelligence provides powerful tools for predicting clinical toxicity and simulating in vivo process [13]. Second, compared to notable preclinical success, clinical outcomes of cancer therapy based on NDDSs are far from satisfied. Curative efficacies of NDDSs on tumors are affected by complex and unknown physiological factors those are highly heterogeneous among individual cases [131]. The lack of animal experimental models which can accurately simulate human cancers resulted in the inconsistent results between in animal models and in patients [132]. Developing models mimicking the heterogeneity of human tumor such as human tumor xenograft models, humanized mouse models, and genetically engineered mouse models may promote the clinical translation of nanomedicine [94]. Moreover, passive tumor-targeting strategies based on EPR effects oversimplify the physiological barriers in the tumor microenvironment. Precise delivery into certain groups of cells or subcellular units should be aimed at [133]. Third, the difficulty of producing NDDSs in large scale is a significant hurdle on the approach to clinical use. The manufacturing process is expected to be simplified and modularized. Incorporating too complex multifunctional elements is not conducive to the large-scale production and the approval procedure for drug registration. Successful clinical translation of nanomedicines could be realized only if the designs of NDDSs are clinical demandoriented [134].

In the future, combining knowledges of engineering, immunology, microbiology and oncology might inspire promising approaches to treating metastatic breast cancer. For instance, the roles played by microbes in cancer progression have been paid more attention to in recent years [135]. Recently, it was reported that enterotoxigenic Bacteroides fragilis (ETBF) could colonize in gut and mammary gland of mice and induce outgrowth and metastasis of breast cancer cells, while non-toxigenic Bacteroides fragilis did not exhibit pro-tumoral and pro-metastatic effects [136]. Microbiome-breast cancer research may help identify specific microbiota members contributing to the pathogenesis of breast cancer and find new treatment regimens. Studying the biological properties of microbes and the interaction between pro-tumoral bacteria and cancer progression also lay the foundation for exploiting the medical potential of bacteria for tumor-targeting drug delivery [137]. Moreover, a variety of NDDSs can be constructed with micro-organismsmodulating materials to regulate gut microbiota, thus enhance systematic antitumor immune response [138, 139].
Although related research is still in its infancy, it is believed that interdisciplinary studies will accelerate the development of NDDSs for metastatic breast cancer therapy. Considering that the predicament of drug delivery exists in various types of cancers, the application of NDDSs in other cancers also has shown great potential and is worth comprehensive investigation.

Research funding: National Natural Science Foundation of China (81871471, 81630052, 81521005, and 81690265), Natural Science Foundation of Shanghai (19ZR1479900), International Partnership Program of CAS (153631KY SB20190013), Natural Science Foundation of Shandong (ZR2019ZD25), Special Research Assistant Project of CAS, China Postdoctoral Science Foundation (2020M681428) and Shanghai Post-doctoral Excellence Program are gratefully acknowledged for financial support.

Author contributions: All authors have accepted responsibility for the entire content of this manuscript and approved its submission.

Competing interests: Authors state no conflict of interest. Informed consent: Informed consent was obtained from all individuals included in this study.

Ethical approval: The local Institutional Review Board deemed the study exempt from review.

\section{References}

1. Siegel RL, Miller KD, Jemal A. Cancer statistics, 2020. CA Cancer J Clin 2020;70:7-30.

2. Steeg PS. Targeting metastasis. Nat Rev Cancer 2016;16:201-18.

3. Wan L, Pantel K, Kang Y. Tumor metastasis: moving new biological insights into the clinic. Nat Med 2013;19:1450-64.

4. Zhuang X, Zhang H, Li X, Li X, Cong M, Peng F, et al. Differential effects on lung and bone metastasis of breast cancer by Wnt signalling inhibitor DKK1. Nat Cell Biol 2017;19:1274-85.

5. Minn AJ, Gupta GP, Siegel PM, Bos PD, Shu W, Giri DD, et al. Genes that mediate breast cancer metastasis to lung. Nature 2005;436: 518-24.

6. Lambert AW, Pattabiraman DR, Weinberg RA. Emerging biological principles of metastasis. Cell 2017;168:670-91.

7. Schroeder A, Heller DA, Winslow MM, Dahlman JE, Pratt GW, Langer R, et al. Treating metastatic cancer with nanotechnology. Nat Rev Cancer 2011;12:39-50.

8. Shi J, Kantoff PW, Wooster R, Farokhzad OC. Cancer nanomedicine: progress, challenges and opportunities. Nat Rev Cancer 2017;17:20-37.

9. Zhang P, Zhai Y, Cai Y, Zhao Y, Li Y. Nanomedicine-based immunotherapy for the treatment of cancer metastasis. Adv Mater 2019;31:1904156.

10. Gonzalez-Valdivieso J, Girotti A, Schneider J, Arias FJ. Advanced nanomedicine and cancer: challenges and opportunities in clinical translation. Int J Pharm 2021;599:120438. 
11. Gadekar V, Borade Y, Kannaujia S, Rajpoot K, Anup N, Tambe V, et al. Nanomedicines accessible in the market for clinical interventions. J Control Release 2021;330:372-97.

12. Lu W, Yao J, Zhu X, Qi Y. Nanomedicines: redefining traditional medicine. Biomed Pharmacother 2021;134:111103.

13. Sindhwani S, Chan WCW. Nanotechnology for modern medicine: next step towards clinical translation. J Intern Med 2021, https:// doi.org/10.1111/joim.13254.

14. Haider N, Fatima S, Taha M, Rizwanullah M, Firdous J, Ahmad R, et al. Nanomedicines in diagnosis and treatment of cancer: an update. Curr Pharm Des 2020;26:1216-31.

15. Koudelka Š, Turánek J. Liposomal paclitaxel formulations. J Control Release 2012;163:322-34.

16. Bukowski R, Ernstoff MS, Gore ME, Nemunaitis JJ, Amato R, Gupta $\mathrm{SK}$, et al. Pegylated interferon alfa- $2 \mathrm{~b}$ treatment for patients with solid tumors: a phase I/II study. J Clin Oncol 2002;20:3841-9.

17. Zhang Z, Wang H, Tan T, Li J, Wang Z, Li Y. Rational design of nanoparticles with deep tumor penetration for effective treatment of tumor metastasis. Adv Funct Mater 2018;28: 1801840

18. Liu X, Wang D, Zhang P, Li Y. Recent advances in nanosized drug delivery systems for overcoming the barriers to anti-PD immunotherapy of cancer. Nano Today 2019;29:100801.

19. Sun H, Su J, Meng Q, Yin Q, Chen L, Gu W, et al. Cancer-cellbiomimetic nanoparticles for targeted therapy of homotypic tumors. Adv Mater 2016;28:9581-8.

20. Gong X, Li J, Tan T, Wang Z, Wang H, Wang Y, et al. Emerging approaches of cell-based nanosystems to target cancer metastasis. Adv Funct Mater 2019;29:1903441.

21. Cao H, Zhang Z, Zhao S, He X, Yu H, Yin Q, et al. Hydrophobic interaction mediating self-assembled nanoparticles of succinobucol suppress lung metastasis of breast cancer by inhibition of VCAM-1 expression. J Control Release 2015;205: 162-71.

22. Wang T, Wang D, Yu H, Feng B, Zhou F, Zhang H, et al. A cancer vaccine-mediated postoperative immunotherapy for recurrent and metastatic tumors. Nat Commun 2018;9:1532.

23. Feng B, Zhou F, Lu W, Wang D, Wang T, Luo C, et al. Phospholipidmimic oxaliplatin prodrug liposome for treatment of the metastatic triple negative breast cancer. Biomater Sci 2017;5: 1522-5.

24. Tang S, Meng Q, Sun H, Su J, Yin Q, Zhang Z, et al. Dual $\mathrm{pH}$-sensitive micelles with charge-switch for controlling cellular uptake and drug release to treat metastatic breast cancer. Biomaterials 2017;114:44-53.

25. Tang S, Meng Q, Sun H, Su J, Yin Q, Zhang Z, et al. Tumormicroenvironment-adaptive nanoparticles codeliver paclitaxel and siRNA to inhibit growth and lung metastasis of breast cancer. Adv Funct Mater 2016;26:6033-46.

26. Xiao J, Duan X, Yin Q, Miao Z, Yu H, Chen C, et al. The inhibition of metastasis and growth of breast cancer by blocking the NF-KB signaling pathway using bioreducible PEI-based/p65 shRNA complex nanoparticles. Biomaterials 2013;34:5381-90.

27. Meng Q, Meng J, Ran W, Wang J, Zhai Y, Zhang P, et al. Lightactivated core-shell nanoparticles for spatiotemporally specific treatment of metastatic triple-negative breast cancer. ACS Nano 2018;12:2789-802.

28. Feng B, Xu Z, Zhou F, Yu H, Sun Q, Wang D, et al. Near infrared light-actuated gold nanorods with cisplatin-polypeptide wrapping for targeted therapy of triple negative breast cancer. Nanoscale 2015;7:14854-64.

29. He X, Cao H, Wang H, Tan T, Yu H, Zhang P, et al. Inflammatory monocytes loading protease-sensitive nanoparticles enable lung metastasis targeting and intelligent drug release for antimetastasis therapy. Nano Lett 2017;17:5546-54.

30. Cao H, Wang H, He X, Tan T, Hu H, Wang Z, et al. Bioengineered macrophages can responsively transform into nanovesicles to target lung metastasis. Nano Lett 2018;18:4762-70.

31. Su J, Sun H, Meng Q, Yin Q, Zhang P, Zhang Z, et al. Bioinspired nanoparticles with NIR-controlled drug release for synergetic chemophotothermal therapy of metastatic breast cancer. Adv Funct Mater 2016;26:7495-506.

32. Thiery JP. Epithelial-mesenchymal transitions in tumour progression. Nat Rev Cancer 2002;2:442-54.

33. Blanco E, Shen H, Ferrari M. Principles of nanoparticle design for overcoming biological barriers to drug delivery. Nat Biotechnol 2015;33:941-51.

34. Li Z, Shan X, Chen Z, Gao N, Zeng W, Zeng X, et al. Applications of surface modification technologies in nanomedicine for deep tumor penetration. Adv Sci 2020;8:2002589.

35. Eckhardt BL, Francis PA, Parker BS, Anderson RL. Strategies for the discovery and development of therapies for metastatic breast cancer. Nat Rev Drug Discov 2012;11:479-97.

36. Semenza GL. The hypoxic tumor microenvironment: a driving force for breast cancer progression. Biochim Biophys Acta 2016; 1863:382-91.

37. Kessenbrock K, Plaks V, Werb Z. Matrix metalloproteinases: regulators of the tumor microenvironment. Cell 2010;141:52-67.

38. Liu Y, Lang T, Zheng Z, Cheng H, Huang X, Wang G, et al. In vivo environment-adaptive nanocomplex with tumor cell-specific cytotoxicity enhances $T$ cells infiltration and improves cancer therapy. Small 2019;15:1902822.

39. Vlodavsky I, Friedmann Y, Elkin M, Aingorn H, Atzmon R, IshaiMichaeli R, et al. Mammalian heparanase: gene cloning, expression and function in tumor progression and metastasis. Nat Med 1999;5:793-802.

40. Kuppusamy P, Li H, Ilangovan G, Cardounel AJ, Zweier JL, Yamada K, et al. Noninvasive imaging of tumor redox status and its modification by tissue glutathione levels. Cancer Res 2002;62:307-12.

41. Wang J, Mao W, Lock LL, Tang J, Sui M, Sun W, et al. The role of micelle size in tumor accumulation, penetration, and treatment. ACS Nano 2015;9:7195-206.

42. Li L, Liu T, Fu C, Meng X, Liu H. Size effect of mesoporous and hollow silica nanoparticles on solid tumor targeting and penetration. J Nanosci Nanotechnol 2016;16:6766-72.

43. Tang L, Gabrielson NP, Uckun FM, Fan TM, Cheng J. Size-dependent tumor penetration and in vivo efficacy of monodisperse drug-silica nanoconjugates. Mol Pharm 2013;10:883-92.

44. Lang T, Dong X, Zheng Z, Liu Y, Wang G, Yin Q, et al. Tumor microenvironment-responsive docetaxel-loaded micelle combats metastatic breast cancer. Sci Bull 2019;64:91-100.

45. Zhang L, Wang Y, Yang D, Huang W, Hao P, Feng S, et al. Shape effect of nanoparticles on tumor penetration in monolayers versus spheroids. Mol Pharm 2019;16:2902-11.

46. Dias DR, Moreira AF, Correia IJ. The effect of the shape of gold core-mesoporous silica shell nanoparticles on the cellular behavior and tumor spheroid penetration. J Mater Chem B 2016; 4:7630-40. 
47. Kolhar P, Anselmo AC, Gupta V, Pant K, Prabhakarpandian B, Ruoslahti E, et al. Using shape effects to target antibody-coated nanoparticles to lung and brain endothelium. Proc Natl Acad Sci U S A 2013;110:10753-8.

48. Geng Y, Dalhaimer P, Cai S, Tsai R, Tewari M, Minko T, et al. Shape effects of filaments versus spherical particles in flow and drug delivery. Nat Nanotechnol 2007;2:249-55.

49. He X, Yu H, Bao X, Cao H, Yin Q, Zhang Z, et al. pH-responsive wormlike micelles with sequential metastasis targeting inhibit lung metastasis of breast cancer. Adv Health Mater 2016;5: 439-48.

50. Mi P, Cabral H, Kataoka K. Ligand-installed nanocarriers toward precision therapy. Adv Mater 2020;32:1902604.

51. Fernandez M, Javaid F, Chudasama V. Advances in targeting the folate receptor in the treatment/imaging of cancers. Chem Sci 2018;9:790-810.

52. Wang LS, Wu LC, Lu SY, Chang LL, Teng IT, Yang CM, et al. Biofunctionalized phospholipid-capped mesoporous silica nanoshuttles for targeted drug delivery: improved water suspensibility and decreased nonspecific protein binding. ACS Nano 2010;4:4371-9.

53. Hu H, Wang J, Wang H, Tan T, Li J, Wang Z, et al. Cell-penetrating peptide-based nanovehicles potentiate lymph metastasis targeting and deep penetration for anti-metastasis therapy. Theranostics 2018;8:3597-610.

54. Ruoslahti E. Tumor penetrating peptides for improved drug delivery. Adv Drug Deliv Rev 2017;110-111:3-12.

55. Wang T, Wang D, Liu J, Feng B, Zhou F, Zhang H, et al. Aciditytriggered ligand-presenting nanoparticles to overcome sequential drug delivery barriers to tumors. Nano Lett 2017;17: 5429-36.

56. Lang T, Liu Y, Zheng Z, Ran W, Zhai Y, Yin Q, et al. Cocktail strategy based on spatio-temporally controlled nano device improves therapy of breast cancer. Adv Mater 2019;31:1806202.

57. Huang Y, Goel S, Duda DG, Fukumura D, Jain RK. Vascular normalization as an emerging strategy to enhance cancer immunotherapy. Cancer Res 2013;73:2943-8.

58. Heldin C-H, Rubin K, Pietras K, Östman A. High interstitial fluid pressure - an obstacle in cancer therapy. Nat Rev Cancer 2004;4: 806-13.

59. Insua-Rodriguez J, Oskarsson T. The extracellular matrix in breast cancer. Adv Drug Deliv Rev 2016;97:41-55.

60. Kalluri R. The biology and function of fibroblasts in cancer. Nat Rev Cancer 2016;16:582-98.

61. Hu C, Liu X, Ran W, Meng J, Zhai Y, Zhang P, et al. Regulating cancer associated fibroblasts with losartan-loaded injectable peptide hydrogel to potentiate chemotherapy in inhibiting growth and lung metastasis of triple negative breast cancer. Biomaterials 2017;144:60-72.

62. Feng B, Hou B, Xu Z, Saeed M, Yu H, Li Y. Self-amplified drug delivery with light-inducible nanocargoes to enhance cancer immunotherapy. Adv Mater 2019;31:1902960.

63. Sun H, Su J, Meng Q, Yin Q, Chen L, Gu W, et al. Cancer cell membrane-coated gold nanocages with hyperthermia-triggered drug release and homotypic target inhibit growth and metastasis of breast cancer. Adv Funct Mater 2017;27:1604300.

64. Lang T, Dong X, Huang Y, Ran W, Yin Q, Zhang P, et al. Ly6C $\mathrm{C}^{\text {hi }}$ monocytes delivering $\mathrm{pH}$-sensitive micelle loading paclitaxel improve targeting therapy of metastatic breast cancer. Adv Funct Mater 2017;27:1701093.
65. Yin J, Lang T, Cun D, Zheng Z, Huang Y, Yin Q, et al. pH-sensitive nano-complexes overcome drug resistance and inhibit metastasis of breast cancer by silencing Akt expression. Theranostics 2017;7:4204-16.

66. Yu H, Guo C, Feng B, Liu J, Chen X, Wang D, et al. Triple-layered phresponsive micelleplexes loaded with sirna and cisplatin prodrug for NF-KB targeted treatment of metastatic breast cancer. Theranostics 2016;6:14-27.

67. Dan Z, Cao H, He X, Zhang Z, Zou L, Zeng L, et al. A pH-responsive host-guest nanosystem loading succinobucol suppresses lung metastasis of breast cancer. Theranostics 2016;6:435-45.

68. Zheng Z, Lang T, Huang X, Wang G, Lee RJ, Teng L, et al. Calcitriolloaded dual-pH-sensitive micelle counteracts pro-metastasis effect of paclitaxel in triple-negative breast cancer therapy. Adv Health Mater 2020;9:2000392.

69. He B, Tan T, Wang H, Hu H, Wang Z, Wang J, et al. Rational design of tumor microenvironment-activated micelles for programed targeting of breast cancer metastasis. Adv Funct Mater 2018;28: 1705622.

70. Xu P, Meng Q, Sun H, Yin Q, Yu H, Zhang Z, et al. Shrapnel nanoparticles loading docetaxel inhibit metastasis and growth of breast cancer. Biomaterials 2015;64:10-20.

71. Feng B, Zhou F, Hou B, Wang D, Wang T, Fu Y, et al. Binary cooperative prodrug nanoparticles improve immunotherapy by synergistically modulating immune tumor microenvironment. Adv Mater 2018;30:1803001.

72. Zhou F, Feng B, Wang T, Wang D, Cui Z, Wang S, et al. Theranostic prodrug vesicles for reactive oxygen species-triggered ultrafast drug release and local-regional therapy of metastatic triplenegative breast cancer. Adv Funct Mater 2017;27:1703647.

73. Feng B, Niu Z, Hou B, Zhou L, Li Y, Yu H. Enhancing triple negative breast cancer immunotherapy by ICG-templated self-assembly of paclitaxel nanoparticles. Adv Funct Mater 2019;30, https://doi. org/10.1002/adfm.201906605.

74. Zhai Y, Ran W, Su J, Lang T, Meng J, Wang G, et al. Traceable bioinspired nanoparticle for the treatment of metastatic breast cancer via DIR-trigged intracellular delivery of methylene blue and cisplatin. Adv Mater 2018:1802378, https://doi.org/10. 1002/adma.201802378.

75. Cao H, Dan Z, He X, Zhang Z, Yu H, Yin Q, et al. Liposomes coated with isolated macrophage membrane can target lung metastasis of breast cancer. ACS Nano 2016;10:7738-48.

76. Tan $\mathrm{T}$, Wang $\mathrm{H}$, Cao H, Zeng L, Wang Y, Wang Z, et al. Deep tumorpenetrated nanocages improve accessibility to cancer stem cells for photothermal-chemotherapy of breast cancer metastasis. Adv Sci 2018;5:1801012.

77. Cao H, Zou L, He B, Zeng L, Huang Y, Yu H, et al. Albumin biomimetic nanocorona improves tumor targeting and penetration for synergistic therapy of metastatic breast cancer. Adv Funct Mater 2017;27:1605679.

78. Tan T, Hu H, Wang H, Li J, Wang Z, Wang J, et al. Bioinspired lipoproteins-mediated photothermia remodels tumor stroma to improve cancer cell accessibility of second nanoparticles. Nat Commun 2019;10:3322.

79. Yin J, Cao H, Wang H, Sun K, Li Y, Zhang Z. Phospholipid membrane-decorated deep-penetrated nanocatalase relieve tumor hypoxia to enhance chemo-photodynamic therapy. Acta Pharm Sin B 2020;10:2246-57.

80. Wang D, Xu Z, Yu H, Chen X, Feng B, Cui Z, et al. Treatment of metastatic breast cancer by combination of chemotherapy and 
photothermal ablation using doxorubicin-loaded DNA wrapped gold nanorods. Biomaterials 2014;35:8374-84.

81. Wu M, Meng Q, Chen Y, Xu P, Zhang S, Li Y, et al. Ultrasmall confined iron oxide nanoparticle MSNs as a $\mathrm{pH}$-responsive theranostic platform. Adv Funct Mater 2014;24:4273-83.

82. Hu J, Zhang G, Ge Z, Liu S. Stimuli-responsive tertiary amine methacrylate-based block copolymers: synthesis, supramolecular self-assembly and functional applications. Prog Polym Sci 2014;39:1096-143.

83. Li H, Huang F, Fan L, Jiang Y, Wang X, Li J, et al. Phosphatidylethanolamine-binding protein 4 is associated with breast cancer metastasis through Src-mediated Akt tyrosine phosphorylation. Oncogene 2014;33:4589-98.

84. Gdowski A, Panchoo M, Treuren TV, Basu A. Emerging therapeutics for targeting Akt in cancer. Front Biosci (Landmark Ed) 2016;21:757-68.

85. Dong XY, Lang TQ, Yin Q, Zhang PC, Li YP. Co-delivery of docetaxel and silibinin using $\mathrm{pH}$-sensitive micelles improves therapy of metastatic breast cancer. Acta Pharmacol Sin 2017;38:1655-62.

86. Nambiar D, Prajapati V, Agarwal R, Singh RP. In vitro and in vivo anticancer efficacy of silibinin against human pancreatic cancer BxBC-3 and PANC-1 cells. Cancer Lett 2013;334:109-17.

87. Hu Q-D, Tang G-P, Chu PK. Cyclodextrin-based host-guest supramolecular nanoparticles for delivery: rom design to applications. Acc Chem Res 2014;47:2017-25.

88. Feldman D, Krishnan AV, Swami S, Giovannucci E, Feldman BJ. The role of vitamin $d$ in reducing cancer risk and progression. Nat Rev Cancer 2014;14:342-57.

89. Cook JA, Gius D, Wink DA, Krishna MC, Russo A, Mitchell JB. Oxidative stress, redox, and the tumor microenvironment. Semi Radiat Oncol 2004;14:259-66.

90. Krysko DV, Garg AD, Kaczmarek A, Krysko O, Agostinis P, Vandenabeele P. Immunogenic cell death and DAMPs in cancer therapy. Nat Rev Cancer 2012;12:860-75.

91. Upputuri PK, Pramanik M. Photoacoustic imaging in the second near-infrared window: a review. J Biomed Opt 2019;24:1-20.

92. Bashkatov AN, Genina EA, Kochubey VI, Tuchin VV. Optical properties of human skin, subcutaneous and mucous tissues in the wavelength range from 400 to $2000 \mathrm{~nm}$. J Phys D Appl Phys 2005;38:2543-55.

93. Feng B, Zhou F, Xu Z, Wang T, Wang D, Liu J, et al. Versatile prodrug nanoparticles for acid-triggered precise imaging and organelle-specific combination cancer therapy. Adv Funct Mater 2016;26:7431-42.

94. Liang C, Xu L, Song G, Liu Z. Emerging nanomedicine approaches fighting tumor metastasis: animal models, metastasis-targeted drug delivery, phototherapy, and immunotherapy. Chem Soc Rev 2016;45:6250-69.

95. Zou L, Wang H, He B, Zeng L, Tan T, Cao H, et al. Current approaches of photothermal therapy in treating cancer metastasis with nanotherapeutics. Theranostics 2016;6:762-72.

96. He B, Hu HY, Tan T, Wang H, Sun KX, Li YP, et al. IR-780-loaded polymeric micelles enhance the efficacy of photothermal therapy in treating breast cancer lymphatic metastasis in mice. Acta Pharmacol Sin 2018;39:132-9.

97. He X, Bao X, Cao H, Zhang Z, Yin Q, Gu W, et al. Tumor-penetrating nanotherapeutics loading a near-infrared probe inhibit growth and metastasis of breast cancer. Adv Funct Mater 2015;25:2831-9.
98. Zhai Y, Su J, Ran W, Zhang P, Yin Q, Zhang Z, et al. Preparation and application of cell membrane-camouflaged nanoparticles for cancer therapy. Theranostics 2017;7:2575-92.

99. Hu CM, Zhang L, Aryal S, Cheung C, Fang RH, Zhang L. Erythrocyte membrane-camouflaged polymeric nanoparticles as a biomimetic delivery platform. Proc Natl Acad Sci U S A 2011; 108:10980-5.

100. Hu CM, Fang RH, Wang KC, Luk BT, Thamphiwatana S, Dehaini D, et al. Nanoparticle biointerfacing by platelet membrane cloaking. Nature 2015;526:118-21.

101. Gao W, Fang RH, Thamphiwatana S, Luk BT, Li J, Angsantikul P, et al. Modulating antibacterial immunity via bacterial membrane-coated nanoparticles. Nano Lett 2015;15:1403-9.

102. Glinsky VV, Glinsky GV, Glinskii OV, Huxley VH, Turk JR, Mossine VV, et al. Intravascular metastatic cancer cell homotypic aggregation at the sites of primary attachment to the endothelium. Cancer Res 2003;63:3805-11.

103. Lee HW, Choi HJ, Ha SJ, Lee KT, Kwon YG. Recruitment of monocytes/macrophages in different tumor microenvironments. Biochim Biophys Acta 2013;1835:170-9.

104. Su J, Sun H, Meng Q, Zhang P, Yin Q, Li Y. Enhanced blood suspensibility and laser-activated tumor-specific drug release of theranostic mesoporous silica nanoparticles by functionalizing with erythrocyte membranes. Theranostics 2017;7:523-37.

105. Boyango I, Barash U, Naroditsky I, Li JP, Hammond E, Ilan N, et al. Heparanase cooperates with RAS to drive breast and skin tumorigenesis. Cancer Res 2014;74:4504-14.

106. Caruana I, Savoldo B, Hoyos V, Weber G, Liu H, Kim ES, et al. Heparanase promotes tumor infiltration and antitumor activity of CAR-redirected T lymphocytes. Nat Med 2015;21:524-9.

107. Lang T, Ran W, Dong X, Zheng Z, Liu Y, Yin Q, et al. Tumor cellsselective bionic nanodevice exploiting heparanase combats metastatic breast cancer. Adv Funct Mater 2018;28:1707289.

108. Su J, Sun H, Meng Q, Yin Q, Tang S, Zhang P, et al. Long circulation red-blood-cell-mimetic nanoparticles with peptideenhanced tumor penetration for simultaneously inhibiting growth and lung metastasis of breast cancer. Adv Funct Mater 2016;26:1243-52.

109. Wang LS, Wu LC, Lu SY, Chang LL, Teng IT, Yang CM, et al. Biofunctionalized phospholipid-capped mesoporous silica nanoshuttles for targeted drug delivery: improved water suspensibility and decreased nonspecific protein binding. ACS Nano 2010;4:4371-9.

110. Fidler IJ. The pathogenesis of cancer metastasis: the 'seed and soil' hypothesis revisited. Nat Rev Cancer 2003;3:453-8.

111. Noy R, Pollard Jeffrey W. Tumor-associated macrophages: from mechanisms to therapy. Immunity 2014;41:49-62.

112. Smith BR, Ghosn EE, Rallapalli H, Prescher JA, Larson T, Herzenberg LA, et al. Selective uptake of single-walled carbon nanotubes by circulating monocytes for enhanced tumour delivery. Nat Nanotechnol 2014;9:481-7.

113. Caso R, Silvera R, Carrio R, Iragavarapu-Charyulu V, GonzalezPerez RR, Torroella-Kouri M. Blood monocytes from mammary tumor-bearing mice: early targets of tumor-induced immune suppression? Int J Oncol 2010;37:891-900.

114. Lang T, Zheng Z, Huang X, Liu Y, Zhai Y, Zhang P, et al. Ternary regulation of tumor microenvironment by heparanase-sensitive micelle-loaded monocytes improves chemo-immunotherapy of 
metastatic breast cancer. Adv Funct Mater 2020:2007402, https://doi.org/10.1002/adfm.202007402.

115. Choi J, Kim HY, Ju EJ, Jung J, Park J, Chung HK, et al. Use of macrophages to deliver therapeutic and imaging contrast agents to tumors. Biomaterials 2012;33:4195-203.

116. Pattabiraman DR, Weinberg RA. Tackling the cancer stem cellswhat challenges do they pose? Nat Rev Drug Discov 2014;13: 497-512.

117. Son S, Song S, Lee SJ, Min S, Kim SA, Yhee JY, et al. Self-crosslinked human serum albumin nanocarriers for systemic delivery of polymerized sirna to tumors. Biomaterials 2013;34:9475-85.

118. Kuai R, Li D, Chen YE, Moon JJ, Schwendeman A. High-density lipoproteins: nature's multifunctional nanoparticles. ACS Nano 2016;10:3015-41.

119. Kadiyala P, Li D, Nunez FM, Altshuler D, Doherty R, Kuai R, et al. High-density lipoprotein-mimicking nanodiscs for chemoimmunotherapy against glioblastoma multiforme. ACS Nano 2019;13:1365-84.

120. Paniker NV, lyer GY. Erythrocyte catalase and detoxication of hydrogen peroxide. Can J Biochem 1965;43:1029-39.

121. Chen YX, Wei CX, Lyu YQ, Chen HZ, Jiang G, Gao XL. Biomimetic drug-delivery systems for the management of brain diseases. Biomater Sci 2020;8:1073-88.

122. Thanuja MY, Anupama C, Ranganath SH. Bioengineered cellular and cell membrane-derived vehicles for actively targeted drug delivery: so near and yet so far. Adv Drug Deliv Rev 2018;132:57-80.

123. Sevencan C, McCoy RSA, Ravisankar P, Liu M, Govindarajan S, Zhu J, et al. Cell membrane nanotherapeutics: from synthesis to applications emerging tools for personalized cancer therapy. Adv Ther 2020;3:1900201.

124. Gao C, Bhattarai P, Zhou Y, Zhang N, Hameed S, Yue X, et al. Harnessing platelets as functional vectors for contrast enhanced ultrasound imaging and fluorescence imaging. RSC Adv 2019;9:41993-9.

125. Alieva M, van Rheenen J, Broekman MLD. Potential impact of invasive surgical procedures on primary tumor growth and metastasis. Clin Exp Metastasis 2018;35:319-31.

126. Liong M, Lu J, Kovochich M, Xia T, Ruehm SG, Nel AE, et al. Multifunctional inorganic nanoparticles for imaging, targeting, and drug delivery. ACS Nano 2008;2:889-96.

127. Zhou Y, Quan G, Wu Q, Zhang X, Niu B, Wu B, et al. Mesoporous silica nanoparticles for drug and gene delivery. Acta Pharm Sin B 2018;8:165-77.
128. Chen Y, Xu P, Chen H, Li Y, Bu W, Shu Z, et al. Colloidal HPMO nanoparticles: silica-etching chemistry tailoring, topological transformation, and nano-biomedical applications. Adv Mater 2013;25:3100-5.

129. Chen Y, Xu P, Wu M, Meng Q, Chen H, Shu Z, et al. Colloidal RBC-shaped, hydrophilic, and hollow mesoporous carbon nanocapsules for highly efficient biomedical engineering. Adv Mater 2014;26:4294-301.

130. Xiao J, Duan X, Yin Q, Zhang Z, Yu H, Li Y. Nanodiamondsmediated doxorubicin nuclear delivery to inhibit lung metastasis of breast cancer. Biomaterials 2013;34: 9648-56.

131. Hare JI, Lammers T, Ashford MB, Puri S, Storm G, Barry ST. Challenges and strategies in anti-cancer nanomedicine development: an industry perspective. Adv Drug Deliv Rev 2017; 108:25-38.

132. Hassanzadeh P, Atyabi F, Dinarvand R. Ignoring the modeling approaches: towards the shadowy paths in nanomedicine. J Control Release 2018;280:58-75.

133. Sun D, Zhou S, Gao W. What went wrong with anticancer nanomedicine design and how to make it right. ACS Nano 2020; 14:12281-90.

134. Guidolin K, Zheng G. Nanomedicines lost in translation. ACS Nano 2019;13:13620-6.

135. Ladaycia A, Loretz B, Passirani C, Lehr CM, Lepeltier E. Microbiota and cancer: in vitro and in vivo models to evaluate nanomedicines. Adv Drug Deliv Rev 2021;170:44-70.

136. Parida S, Wu S, Siddharth S, Wang G, Muniraj N, Nagalingam A, et al. A pro-carcinogenic colon microbe promotes breast tumorigenesis and metastatic progression and concomitantly activates Notch and $\beta$-Catenin axes. Cancer Discov 2021;11: 1-20.

137. Fan JX, Li ZH, Liu XH, Zheng DW, Chen Y, Zhang XZ. Bacteriamediated tumor therapy utilizing photothermally-controlled TNF- $\alpha$ expression via oral administration. Nano Lett $2018 ; 18$ : 2373-80.

138. Zheng DW, Dong X, Pan P, Chen KW, Fan JX, Cheng SX, et al. Phage-guided modulation of the gut microbiota of mouse models of colorectal cancer augments their responses to chemotherapy. Nat Biomed Eng 2019;3:717-28.

139. Zheng DW, Li RQ, An JX, Xie TQ, Han ZY, Xu R, et al. Prebioticsencapsulated probiotic spores regulate gut microbiota and suppress colon cancer. Adv Mater 2020;32:2004529. 